\title{
Cristóbal, la tormenta tropical del 2020 que dejó precipitaciones atípicas en la Península de Yucatán
}

\section{Cristobal, the tropical storm in 2020 that left atypical rainfall in the Yucatan Peninsula}

\author{
María Engracia Hernández Cerda \\ Investigadora del Instituto de Geografía. Universidad Nacional Autónoma de México, México. \\ mehc@unam.mx | 00000-0003-2996-8771 \\ Enrique Azpra Romero \\ Investigador del Centro de Ciencias de la Atmósfera. Universidad Nacional Autónoma de México, México \\ $\underline{\text { ear1@ atmosfera.unam.mx }}$ | 00000-0002-3902-4697 \\ Claudia Tania Lomas Barrié \\ Investigadora del Instituto Nacional de Investigaciones Forestales, Agrícolas y Pecuarias, CIRSE - C.E. Mocochá, \\ Yucatán, México. \\ lomas.claudia@inifap.gob.mx $\mid \underline{0000-0003-1260-607 X}$
}

\begin{abstract}
Para citar este artículo: Lomas Barrié, C. T., Hernández Cerda, M. E. y Azpra Romero. E. (2021). Cristóbal, la tormenta tropical del 2020 que dejó precipitaciones atípicas en la Península de Yucatán. Entorno Geográfico, (21), 125-156. https://doi.org/10.25100/eg.v0i21.11296
\end{abstract}

\section{Resumen}

Los ciclones tropicales en sus diferentes etapas depresión, tormenta y huracán, han sido tema de investigación en las últimas décadas, este trabajo estudia la variabilidad espacial de la precipitación generada por la tormenta tropical Cristóbal en su formación y paso por la Península de Yucatán, México. Esta perturbación, surgió de la depresión tropical número tres a finales de mayo de 2020. Fue declarada tormenta tropical en la Sonda de Campeche el 31 de mayo antes del inicio de la temporada oficial de ciclones del Atlántico. Cristóbal se mantuvo anormalmente estacionario por tres días en el sur del Golfo de México, entrando a tierra en la entidad de Campeche para desplazarse al norte, y terminar impactando en Luisiana, Estados Unidos de América. En la Península de Yucatán provocó precipitaciones diarias que superaron $240 \%$ a la media del mes de junio (110-220 mm), el récord histórico en cuatro estaciones meteorológicas y el acumulado de los seis días alcanzó el 90\% respecto a la media anual en determinadas áreas, lo que dejó daños en la actividad agropecuaria y en la infraestructura urbana. 
Palabras clave: Huracán, Variabilidad climática, Golfo de México

\begin{abstract}
The tropical cyclones on different stages depression, storm and hurricane, have been topics of research in recent decades. This work studies the spatial variability of the precipitation generated by the tropical storm Cristóbal, which, since its formation and passing through the Yucatan Peninsula, Mexico. This tropical disturbance arose in late May from tropical depression number three. Cristóbal was declared as a tropical storm in the Campeche Sonda, Mexico on May 31 before the beginning of the official Atlantic season. Cristobal stayed abnormally for three days in the southern Gulf of Mexico, entering the Campeche entity to move north, and ending up affecting Louisiana, United States as a tropical storm. In the Yucatan Peninsula, daily rainfall exceeded over $240 \%$ of June average $(110-220 \mathrm{~mm})$. The historical record in four meteorological stations and the accumulated rainfall registered in six days represent $90 \%$ of the annual average in certain areas, which damaged agricultural activity and urban infrastructure.
\end{abstract}

Keywords: Hurricane, Climatic Variability, Gulf of Mexico.

Recibido: 24 de octubre del 2020

Aceptado: 02 de febrero del 2021

\title{
Introducción
}

En el Océano Atlántico el $97 \%$ de los ciclones tropicales (CT) se originan entre el 1 de junio y el 30 de noviembre, en un periodo de datos de 1851 a 2013 (Neumann et al., 1993; Landsea et al., 2011; NOAA, 2020a). Sin embargo, pueden generarse fuera de dicho periodo como los reportados en la temporada de 2005 (Landsea, 2007). Por otro lado, en el Pacífico Nororiental la temporada se extiende entre el 15 de mayo y 30 de noviembre (Rosengaus et al., 2014; Villicaña et al., 2016).

Las condiciones necesarias para el origen y desarrollo de un CT son, temperatura de la superficie del océano por arriba de $26^{\circ} \mathrm{C}$, débil cizalladura vertical del viento y una baja presión en los niveles bajos de la atmósfera coincidentes con flujo divergente en los niveles altos, entre otras (Azpra et al., 2001).

Para el año 2020, el pronóstico oficial emitido por la National Oceanic and Atmospheric Administration (NOAA), indicó que habría $60 \%$ por arriba de la actividad normal de ciclones, 
basados en el pronóstico de la Oscilación del Sur y El Niño, ya que este fenómeno influye de forma importante en la actividad de los CT en el océano Atlántico (Tang and Neelin, 2004; NOAA, 2020b), y a otros factores como la cizalladura vertical reducida del viento, vientos alisios tropicales más débiles del Atlántico y un monzón mejorado en África occidental (Lupo et al., 2008; NOAA, 2020c). Por su parte, el Servicio Meteorológico Nacional (SMN) el 20 de mayo publicó su pronóstico para la temporada indicando que éste sería superior a la media (SMN, 2020a).

Entre el 16 y el 19 de mayo del 2020 se presentó el primer sistema con nombre: Arthur; y el segundo fue Bertha entre el 27 y 28 del mismo mes; ambos antes del inicio oficial de la temporada. Cristóbal, sistema del que se ocupa este artículo, alcanzó intensidad de tormenta tropical (TT) el 1 de junio y, el 8 de junio se emitió su último aviso como sistema tropical (NOAA, 2020d).

Los CT se mueven aproximadamente en una trayectoria parabólica. Inicialmente hacia el este, bajo la acción de los vientos alisios, mientras aumentan su latitud, hasta encontrarse a los vientos del oeste, cuando son arrastrados hacia el oeste, siempre aumentando su latitud. Sobre el Atlántico gran cantidad de CT se originan en una franja que se extiende entre 10 y $20^{\circ} \mathrm{N}$ de latitud y entre los 60 y $80^{\circ} \mathrm{W}$, esto ocasiona que la Península de Yucatán se encuentre en la trayectoria de ellos (Azpra et al., 2001; Díaz, 2010).

La Península de Yucatán con una superficie de 141,714.50 km²(INEGI, 2020) se caracteriza por una orografía plana que no sobrepasa los $200 \mathrm{msnm}$, con excepción de una cumbre de 390 msnm (Lugo-Hub et al., 1992), lo que da lugar a que la lluvia sea de tipo convectiva principalmente. Durante la mitad caliente del año el anticiclón de Bermudas-Azores que se localiza en el Atlántico Norte, se desplaza hacia el norte permitiendo la circulación de los vientos alisios, en donde vienen inmersas las ondas del Este, ambos introducen humedad a la Península. En esta época y hasta principios del otoño, esta zona recibe el $47.3 \%$ de CT provenientes del Atlántico (Azpra, et al., 2001).

Los CT han sido ampliamente estudiados a nivel mundial por la pérdida de vidas humanas y que a su paso devastan campos, poblados y ciudades, pero a la par transportan el agua necesaria para las actividades del hombre. En la actualidad se analizan dentro del contexto del calentamiento global, para definir si su frecuencia e intensidad se ha incrementado (Jiménez et al., 2007; UNESCO, 2009; Farfán et al., 2015). 
Los CT se han incluido en el grupo de amenazas hidro-meteorológicas junto con las inundaciones y las sequías por los daños que ocasionan tanto por la variabilidad en su intensidad como por sus trayectorias (Sánchez, 2006; Farfán et al, 2015).

El objetivo de este trabajo es estudiar la distribución espacio temporal de la lluvia y los efectos producidos por la TT Cristóbal, como uno de los sistemas tropicales más tempranos registrados en el Atlántico con impacto en las tres entidades (Campeche, Quintana Roo y Yucatán) que conforman la Península de Yucatán.

\section{Metodología \\ Área de estudio}

La Península de Yucatán se encuentra localizada en el extremo este de la República Mexicana, como se observa en la Figura 1. Con base en la carta de Clima, escala 1: 1000000 (García, 1997), el clima en su componente térmico es cálido, esto es que la temperatura media anual es mayor a $\operatorname{los} 22^{\circ} \mathrm{C}$ y la media del mes más frío de $18^{\circ} \mathrm{C}$; el régimen de humedad es de verano lo que significa que por lo menos llueve diez veces más en el mes más húmedo de la mitad caliente del año que en el más seco, y durante el verano se caracteriza por la presencia de un periodo de sequía intraestival, de medio verano o canícula. El gradiente de humedad se distribuye de mayor a menor de norte a sur y de oriente a poniente. El clima de mayor área es el subhúmedo (Aw) en sus tres variantes de humedad, el cual se diferencia del húmedo (Am) por su índice P/T mayor a 55.3, y que se ubica en extremo suroeste del estado de Campeche y la isla de Cozumel. En la porción noreste sobre la costa del estado de Yucatán, se presentan dos franjas, una árida $\left(\mathrm{BS}_{0}\right)$ y una semiárida $\left(\mathrm{BS}_{1}\right)$.

Con base a las cartas hipsométrica y topográfica del INEGI (2020b y 2019), la orografía de la Península se caracteriza por no sobrepasar en general los $300 \mathrm{~m}$ de altitud, su gradiente altitudinal va de menor a mayor desde las líneas de costa $(0 \mathrm{msnm})$ al centro-sur (hasta $300 \mathrm{msnm})$, este gradiente está definido por la emergencia de la plataforma continental desde el Golfo de México y limitada al sur por el petén de Guatemala y las montañas Maya de Belice, que provoca ondulaciones en el terreno (López, 1973), generando las mayores elevaciones, el cerro Benito Juárez con 210 msnm en el sur de Yucatán, el cerro Champerico de 390 msnm en el sur de Campeche en el límite con Guatemala y cerro El Charro de 230 msnm al sur de Quintana Roo en el límite con Belice. 
Fuente: Elaboración propia con información del INEGI, 2010.

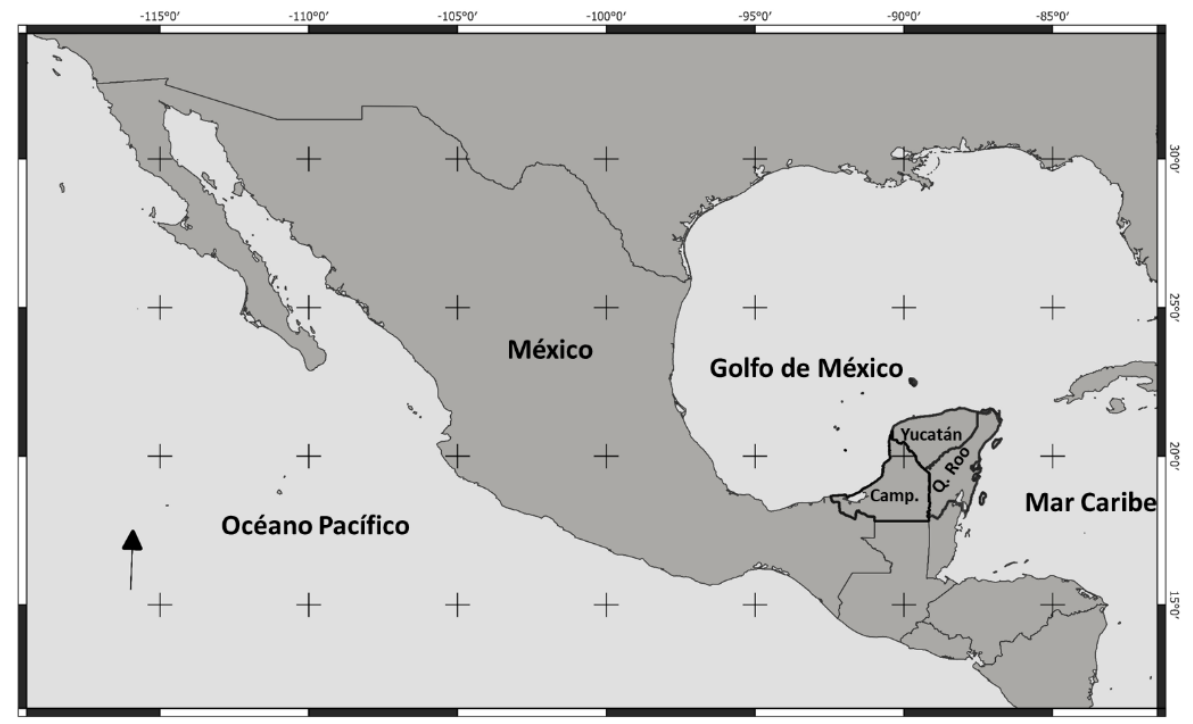

Figura 1. Localización de la Península de Yucatán, entidades que la conforman Campeche, Quintana Roo y Yucatán.

\section{Condiciones climáticas y meteorológicas}

Para este trabajo se obtuvieron y analizaron los datos de precipitación diaria de 235 estaciones climáticas superficiales convencionales y de 7 observatorios, información obtenida del banco nacional de datos climatológicos, del Servicio Meteorológico Nacional (SMN), que depende de la Comisión Nacional del Agua (CONAGUA), la cual forma parte de la Secretaría de Medio Ambiente y Recursos Naturales (SEMARNAT). De las 235 estaciones analizadas, 81, 59 y 31 correspondieron a Campeche, Quintana Roo y a Yucatán (Figura 2).

El periodo histórico de datos climáticos analizado es desde 1951 al 2010, las estaciones contempladas tienen más de 30 años, con al menos 26 días de información por mes y con mínimo 10 meses por año. Para cada una de ellas se calcularon las precipitaciones máximas diarias, las mensuales acumuladas, por periodo de precipitación: el de la mitad caliente del año que se define como el acumulado de mayo a octubre y el periodo de invierno de noviembre a abril, así como la anual acumulada. 
Fuente: Elaboración propia con datos de las estaciones convencionales CONAGUA-SMN, meteorológicas automatizadas de CONAGUA-SMN, INIFAP, UNAM e INEGI, 2010.

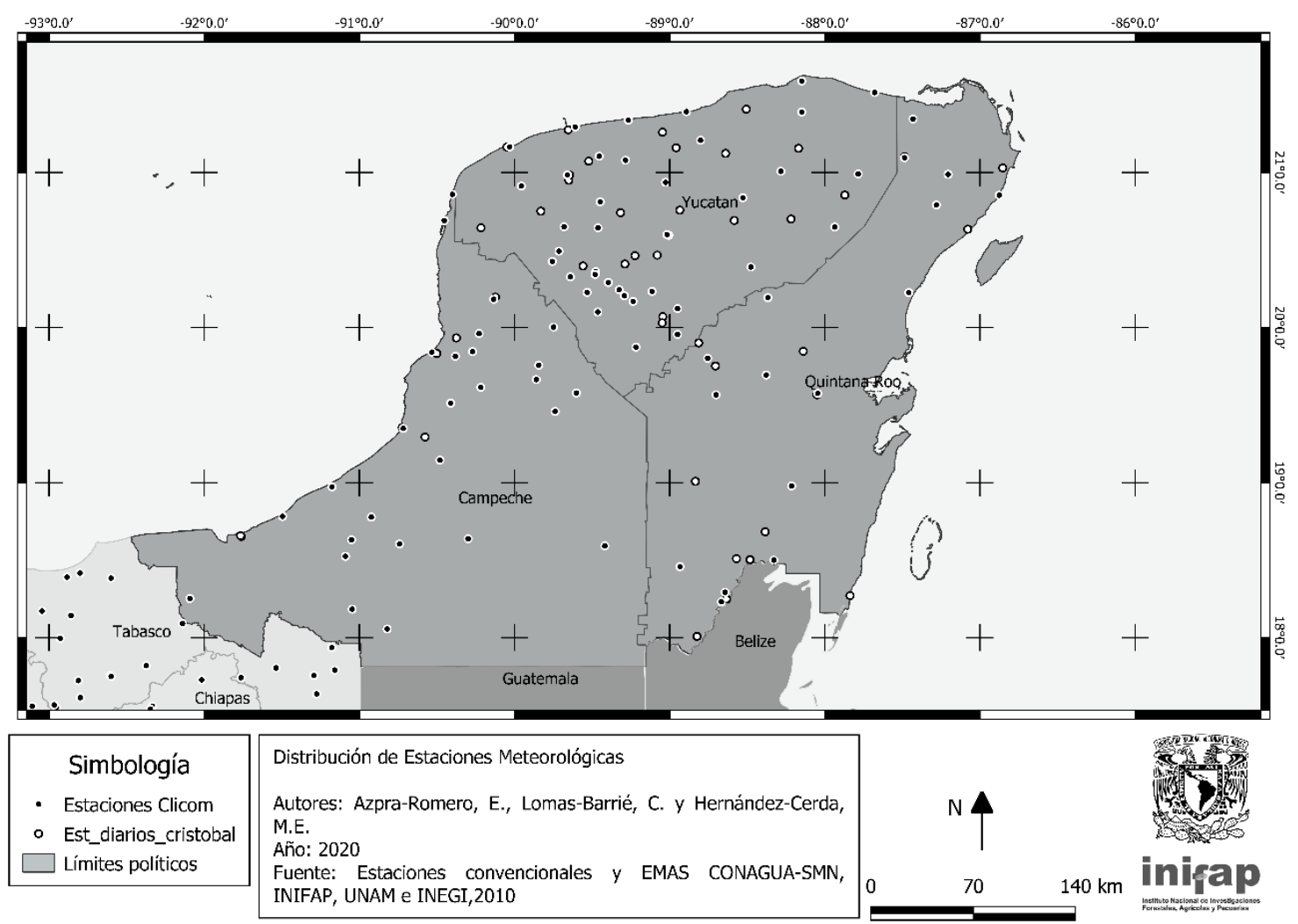

Figura 2. Localización de las estaciones meteorológicas convencionales y automatizadas.

\section{La TT Cristóbal}

Los datos de lluvia diarios durante el periodo del CT del 31 de mayo al 5 de junio de 2020 cuando se localizó sobre la Península de Yucatán, fueron obtenidos directamente de la administración regional de la CONAGUA.

También se utilizaron los datos de las estaciones meteorológicas automatizadas (EMA's) del SMN, del Instituto Nacional de Investigaciones Forestales, Agrícolas y Pecuarias (INIFAP) y de la Universidad Nacional Autónoma de México (UNAM). De estas estaciones se calcularon los datos de precipitación diaria acumulada para el periodo del CT.

Con la información de las estaciones, se generaron mapas con el modelo de interpolación Linear Kriging slope $=1$ y anisotopy=0 en el software Surfer v. 13, los cuales se exportaron a QGIS v. 3.0 para el análisis espacial. Y se evaluó el comportamiento de la precipitación espacio - temporal. 
Cambe mencionar, de acuerdo a Álvarez-Rodríguez (2011 y 2019) que las mayores elevaciones y las pendientes fuertes están estrechamente ligadas con las estaciones con precipitaciones más intensas, sin embargo, el nuevo método de interpolación (Nick, 2010) se sostiene que cuando mayor es la pendiente mayor es la cantidad de precipitación y que, cuando la altitud es menor a $300 \mathrm{msnm}$, la influencia de la orografía no es importante, características que se cumplen en el área de estudio; por lo tanto, para el presente trabajo, no se tomó en cuenta para las interpolaciones.

\section{Condiciones climáticas de los CT vs TT Cristóbal}

Para el análisis de las Depresiones Tropicales (DT), TT y Huracanes (H) que entraron a la Península de Yucatán se utilizaron los datos reportados por el Centro Nacional de Huracanes (NHC) de la NOAA.

\section{Impactos de TT Cristóbal}

Los datos del impacto socio-económico provienen de fuentes de información hemerográfica.

\section{Resultados}

\section{Condiciones climáticas de la Península}

Durante la época invernal (de noviembre a abril), la península recibe lluvia a causa de la llegada de frentes fríos que alcanzan estas latitudes y pueden convertirse en frentes estacionarios, ocasionando que se incremente la posibilidad de lluvia. En la mitad caliente del año, los sistemas meteorológico más importantes que proporcionan precipitación, son los vientos alisios, las ondas tropicales del Este y las perturbaciones tropicales, aunque para la media anual éstos últimos no representan gran aporte (Mosiño y García, 1974; Orellana, et al., 2009), sin embargo, la humedad que depositan aumenta las lluvias convectivas en la región.

Al año, se encontró que se registran precipitaciones menores de 700 mm a más de $1400 \mathrm{~mm}$ al sur y mayor de $1800 \mathrm{~mm}$ al suroeste (Figura 3). La costa noroeste de Yucatán es la región con menor cantidad de precipitación anual con valores inclusive de alrededor de $600 \mathrm{~mm}$ en las inmediaciones de Sisal. Los valores que predominan en la mayor parte del área de estudio están entre 900 a 1200 $\mathrm{mm}$. 
Fuente: Elaboración propia con datos de las estaciones convencionales CONAGUA-SMN, meteorológicas automatizadas de CONAGUA-SMN, INIFAP, UNAM e INEGI,

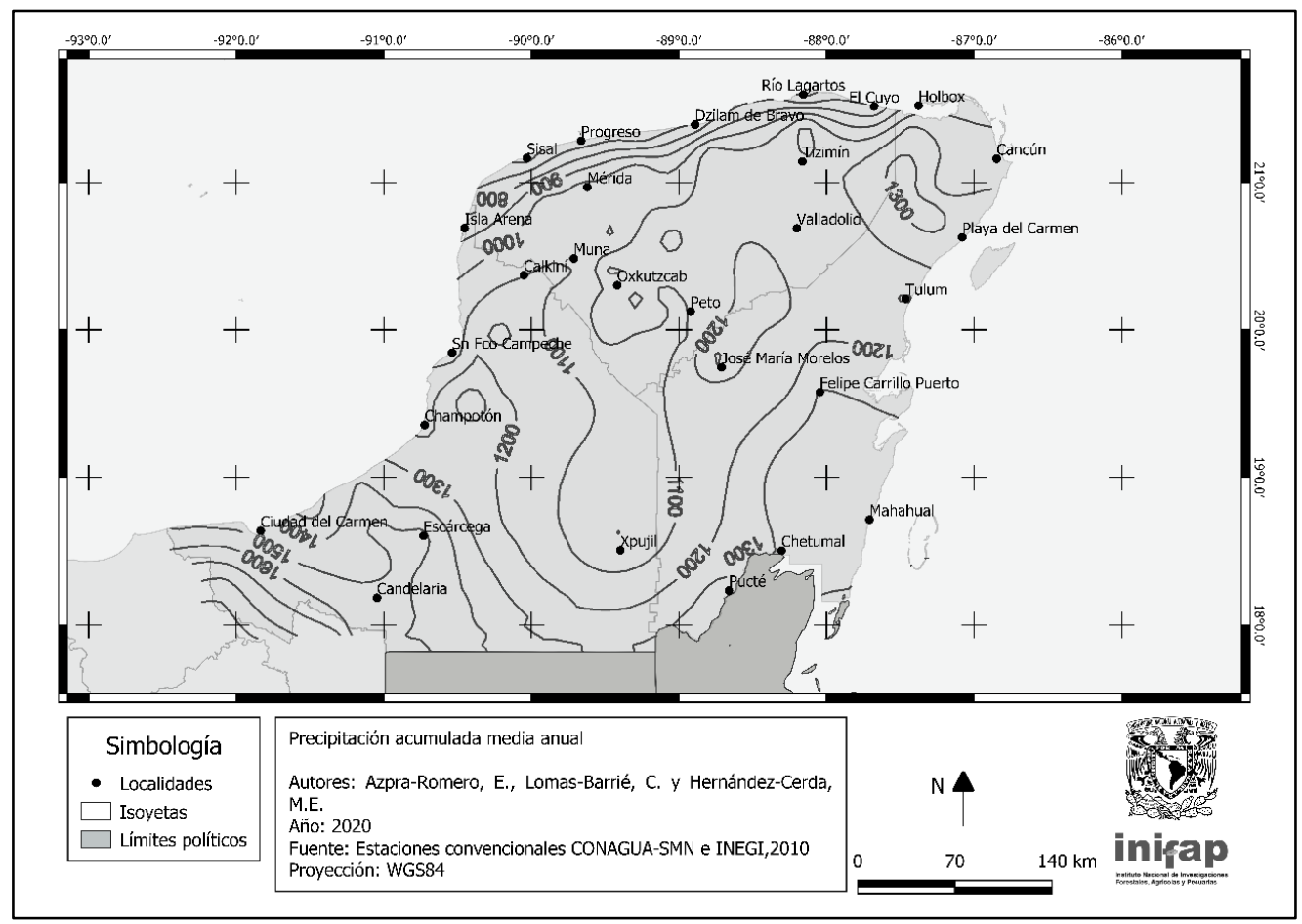

Figura 3. Precipitaciones medias anuales $(\mathrm{mm})$ acumuladas en la Península de Yucatán

La precipitación que se recibe en la mitad caliente del año, de mayo a octubre se muestra en la Figura 4, su distribución es parecida a la distribución anual, la costa norte de la península recibe menor cantidad de precipitación, sólo que, en este caso, los valores están alrededor de 400 mm en el noroeste, y hacia el sur en esta época se alcanzan valores mayores a $1200 \mathrm{~mm}$ y de 1300 al sureste.

De las figuras 5 a la 10, se muestra el comportamiento espacial de la lluvia en los meses de la mitad caliente del año, en el mes de mayo los valores más altos (más de $120 \mathrm{~mm}$ ) se registran al nororiente del estado de Yucatán, menos de $50 \mathrm{~mm}$ en el norte y entre 70 y $90 \mathrm{~mm}$ en el resto de la zona, esto relacionado con el inicio del arribo de las ondas tropicales (OT) que depositan la precipitación en las costas del Caribe (Figura 5).

En el mes de junio la lluvia aumenta, los valores están entre 110 y 190 mm y se registran en la mayor parte de la Península; y los mayores a $200 \mathrm{~mm}$ al oeste de Campeche y los menores de 110 mm en el norte de la Península (Figura 6). 
Fuente: Elaboración propia con datos de las estaciones convencionales CONAGUA-SMN, meteorológicas automatizadas de CONAGUA-SMN, INIFAP, UNAM e INEGI, 2010.

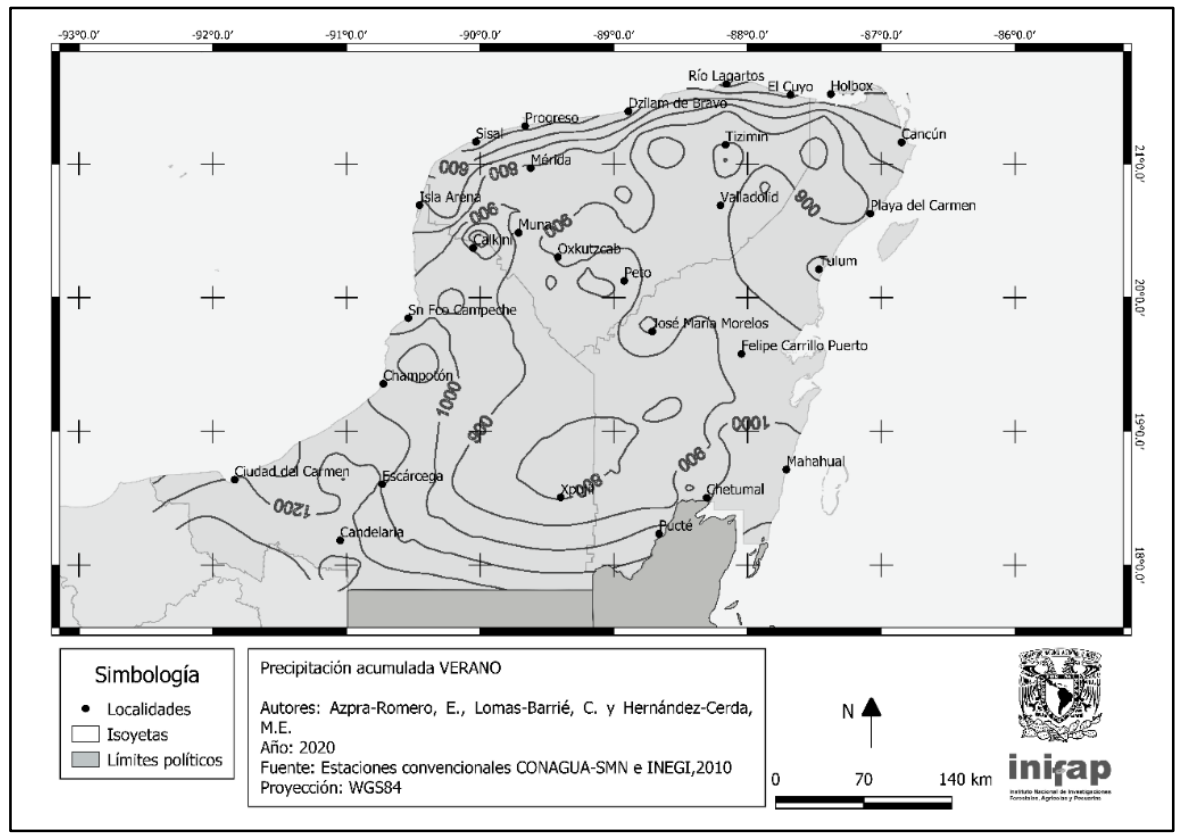

Figura 4. Precipitaciones medias acumuladas $(\mathrm{mm})$ del periodo de la mitad caliente del año (mayo a octubre) en la Península de Yucatán

Fuente: Elaboración propia con datos de las estaciones convencionales CONAGUA-SMN, meteorológicas automatizadas de CONAGUA-SMN, INIFAP, UNAM e INEGI, 2010.

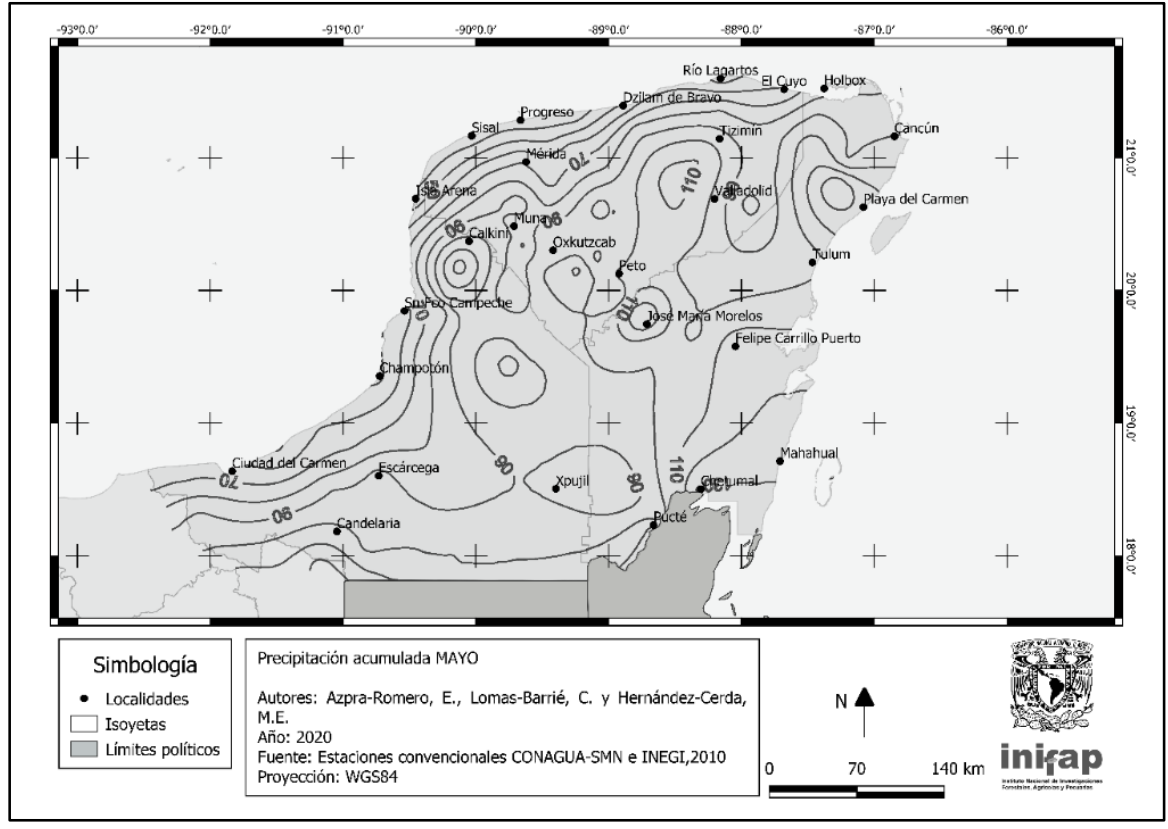

Figura 5. Precipitación media acumulada $(\mathrm{mm})$ del mes de mayo para la Península de Yucatán 
Fuente: Elaboración propia con datos de las estaciones convencionales CONAGUASMN, meteorológicas automatizadas de CONAGUA-SMN, INIFAP, UNAM e INEGI, 2010.

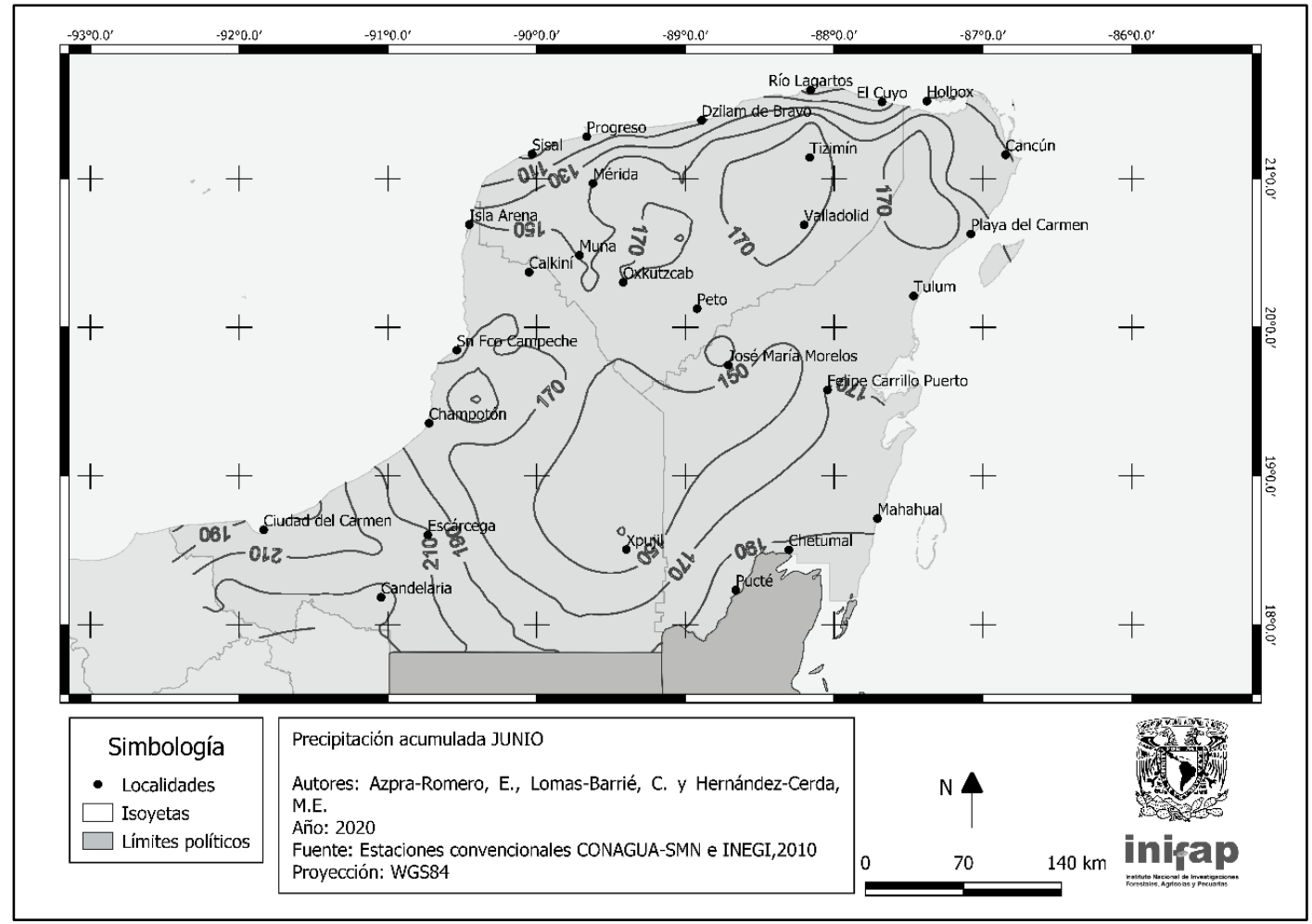

Figura 6. Precipitación media acumulada $(\mathrm{mm})$ del mes de junio para la Península de Yucatán

En julio la lluvia acumulada sufre una disminución respecto a junio, registrándose valores de más de $150 \mathrm{~mm}$ al sur de la Península, más de $230 \mathrm{~mm}$ en el suroeste y menos de $100 \mathrm{~mm}$ en el norte (Figura 7). En la parte centro norte se registran valores entre 130 y $170 \mathrm{~mm}$, esto asociado al fenómeno de canícula o sequía de medio verano, que en la Península se caracteriza por la entrada de los vientos del Este que normalmente arrastran arena del Sahara y disminuyen la actividad convectiva en la Zona Intertropical de Convergencia (ITCZ) y su arribo a la Península (Rosengaus et al., 2014; Small et al., 2007). 
Fuente: Elaboración propia con datos de las estaciones convencionales CONAGUASMN, meteorológicas automatizadas de CONAGUA-SMN, INIFAP, UNAM e INEGI, 2010 .

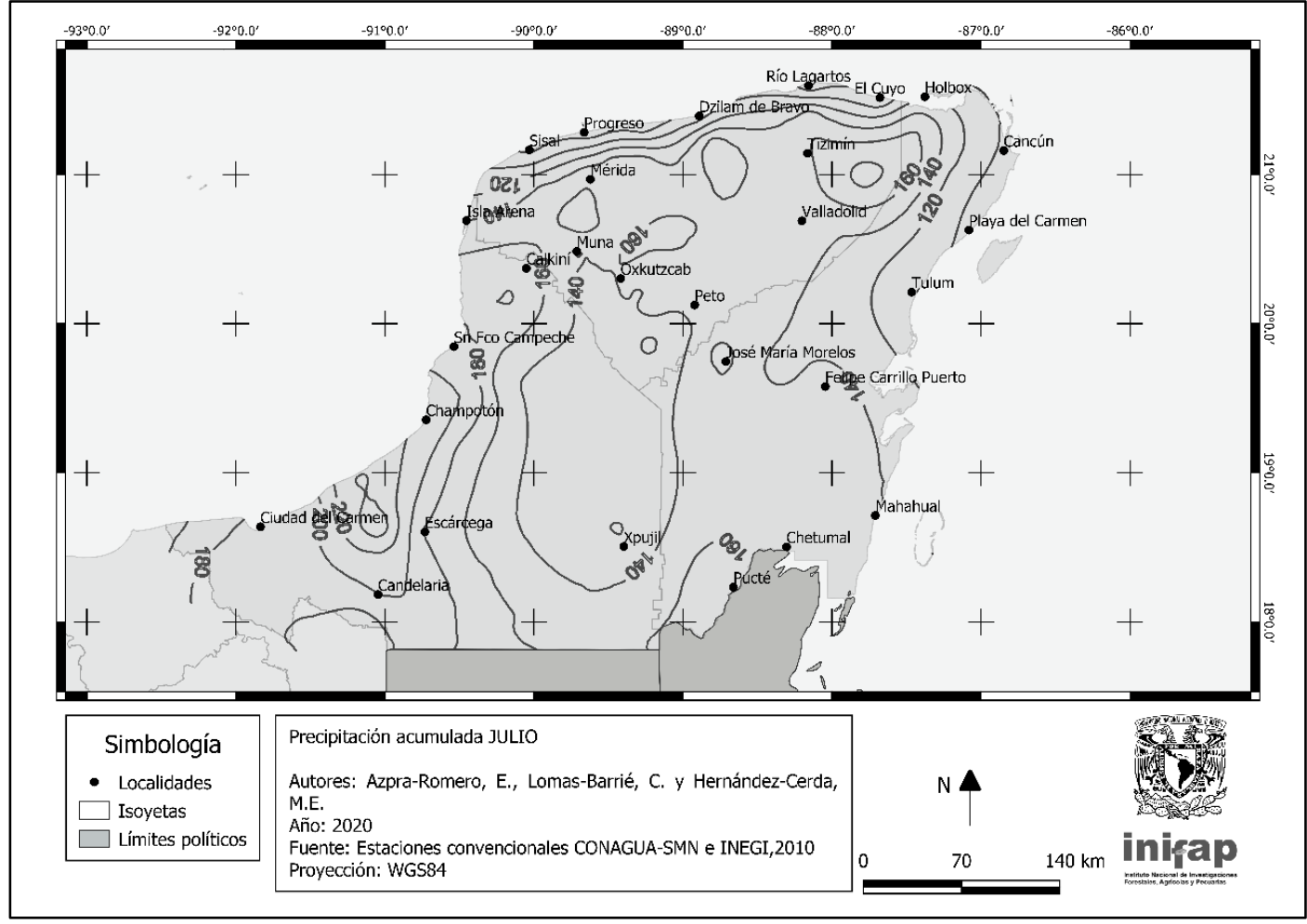

Figura 7. Precipitación media acumulada $(\mathrm{mm})$ del mes de julio para la Península de Yucatán

Durante el mes de agosto predominan los valores entre 160 y $180 \mathrm{~mm}$, en el suroeste mayores de $220 \mathrm{~mm}$ y en el norte y sureste menores de $90 \mathrm{~mm}$ (Figura 8).

En el mes de septiembre se observa el mismo patrón que en agosto, pero ahora las cantidades de lluvia que se reciben en la mayor parte del territorio están entre $180 \mathrm{~mm}$ y $200 \mathrm{~mm}$, sin embargo, las áreas con mayores precipitaciones de $240 \mathrm{~mm}$ se localizan en el suroeste y menos de $120 \mathrm{~mm}$ en el norte (Figura 9), es en este mes donde la actividad de la ITCZ es más intensa y la formación de CT se vuelve más activa (Landsea, 2018).

En octubre empieza a disminuir la lluvia, se registran valores de más de $140 \mathrm{~mm}$ y aún mayores a $160 \mathrm{~mm}$, al sur, oeste y este de la Península. Menos de $120 \mathrm{~mm}$ en el centro y norte de la zona estudiada (Figura 10), ya que se intercalan entre la llegada de las OT y el arribo de los Frentes Fríos. 
Fuente: Elaboración propia con datos de las estaciones convencionales CONAGUASMN, meteorológicas automatizadas de CONAGUA-SMN, INIFAP, UNAM e INEGI, 2010.

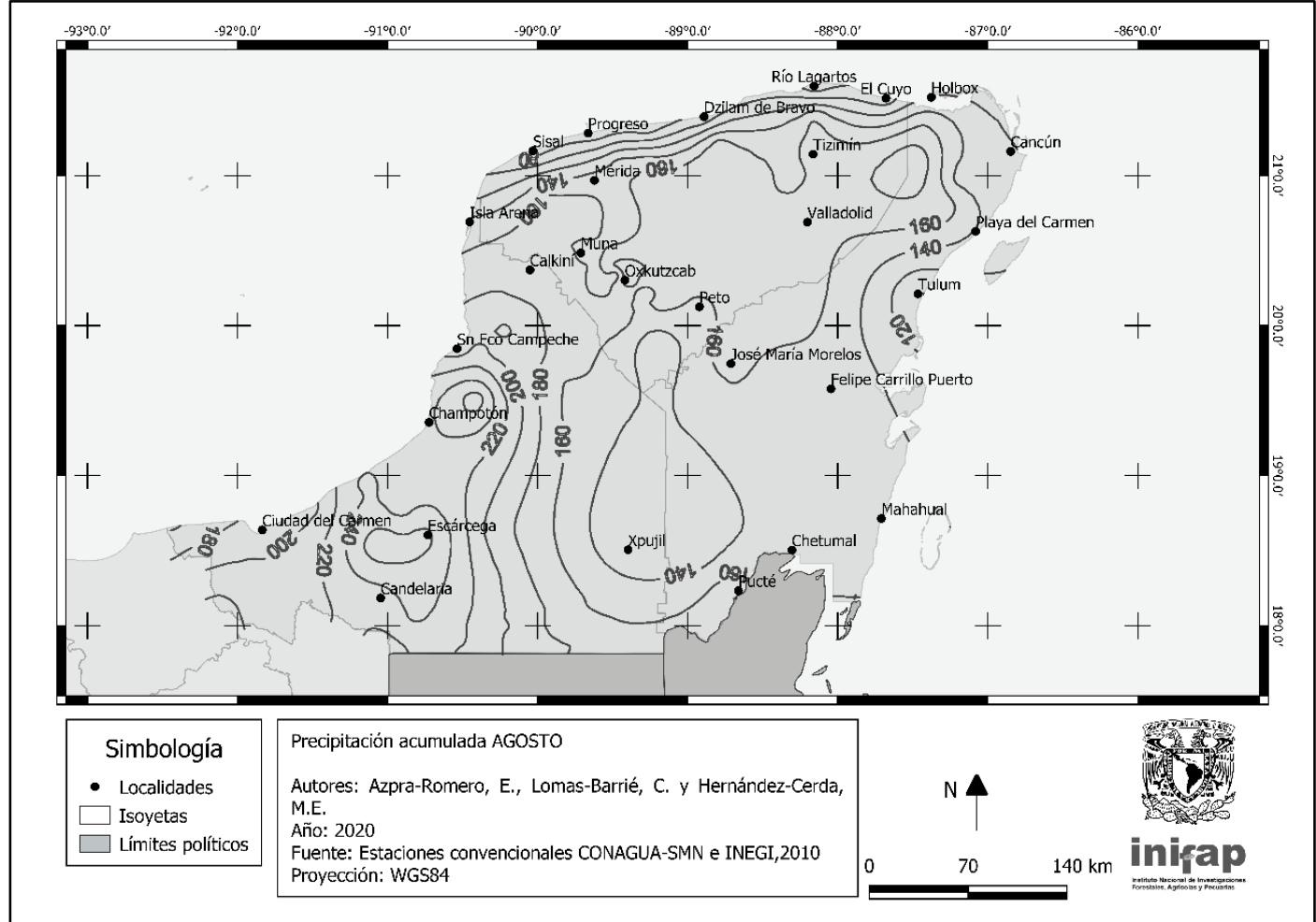

Figura 8. Precipitación media acumulada $(\mathrm{mm})$ del mes de agosto para la Península de Yucatán

El mes más lluvioso de la temporada húmeda es septiembre, las lluvias empiezan a aumentar desde mayo progresivamente hasta el máximo en septiembre, cuando empiezan a decaer.

En cuanto al análisis de los valores de las precipitaciones máximas diarias del periodo estudiado (de 1951 a 2010), sólo se consideraron aquellos eventos con valores por arriba de $100 \mathrm{~mm}$ de lluvia (valor que corresponde al mínimo de lluvia acumulada en 6 días por la TT Cristóbal), se analizaron 938 datos para cada estado. En Yucatán, septiembre es el mes que presentó el mayor porcentaje de días (22.9\%). Para Quintana Roo es en el mes de junio (20.8\%) y en Campeche septiembre (23.2\%). 
Fuente: Elaboración propia con datos de las estaciones convencionales CONAGUA-SMN, meteorológicas automatizadas de CONAGUA-SMN, INIFAP, UNAM e INEGI, 2010.

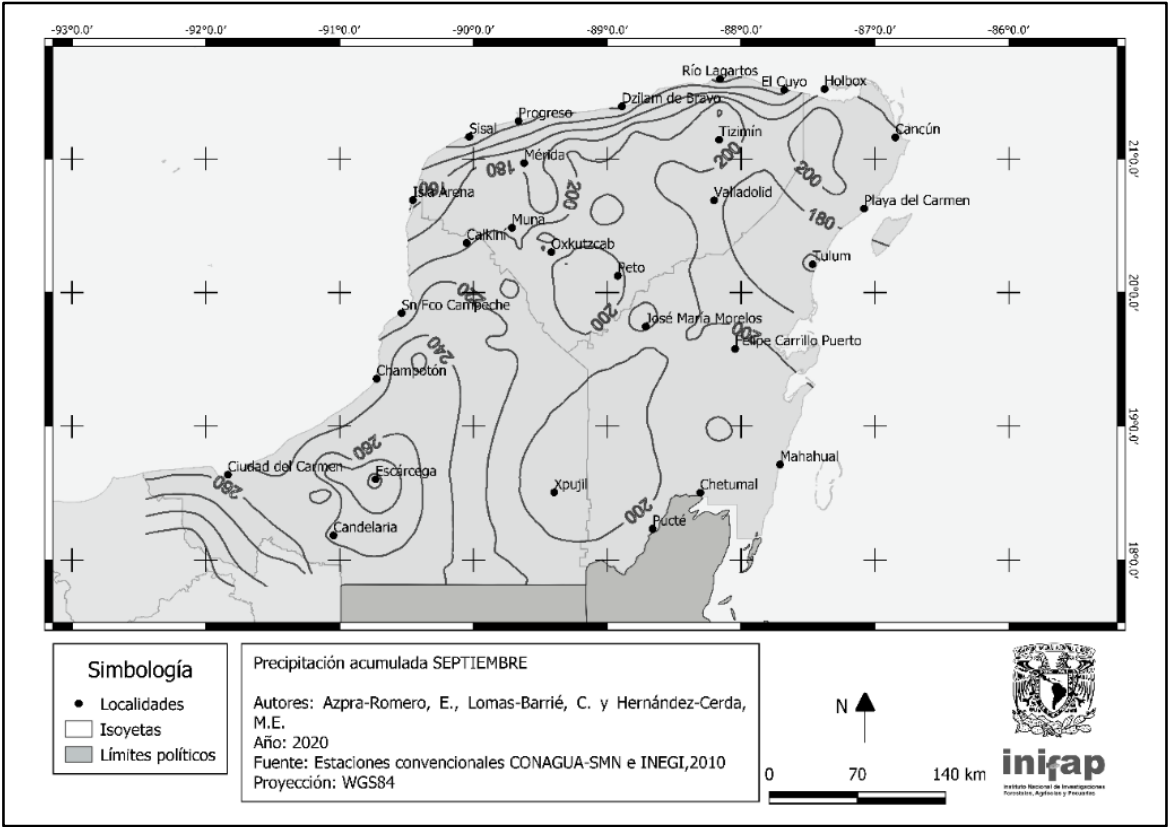

Figura 9. Precipitación media acumulada $(\mathrm{mm})$ del mes de septiembre para la Península de Yucatán.

Fuente: Elaboración propia con datos de las estaciones convencionales CONAGUA-SMN, meteorológicas automatizadas de CONAGUA-SMN, INIFAP, UNAM e INEGI, 2010.

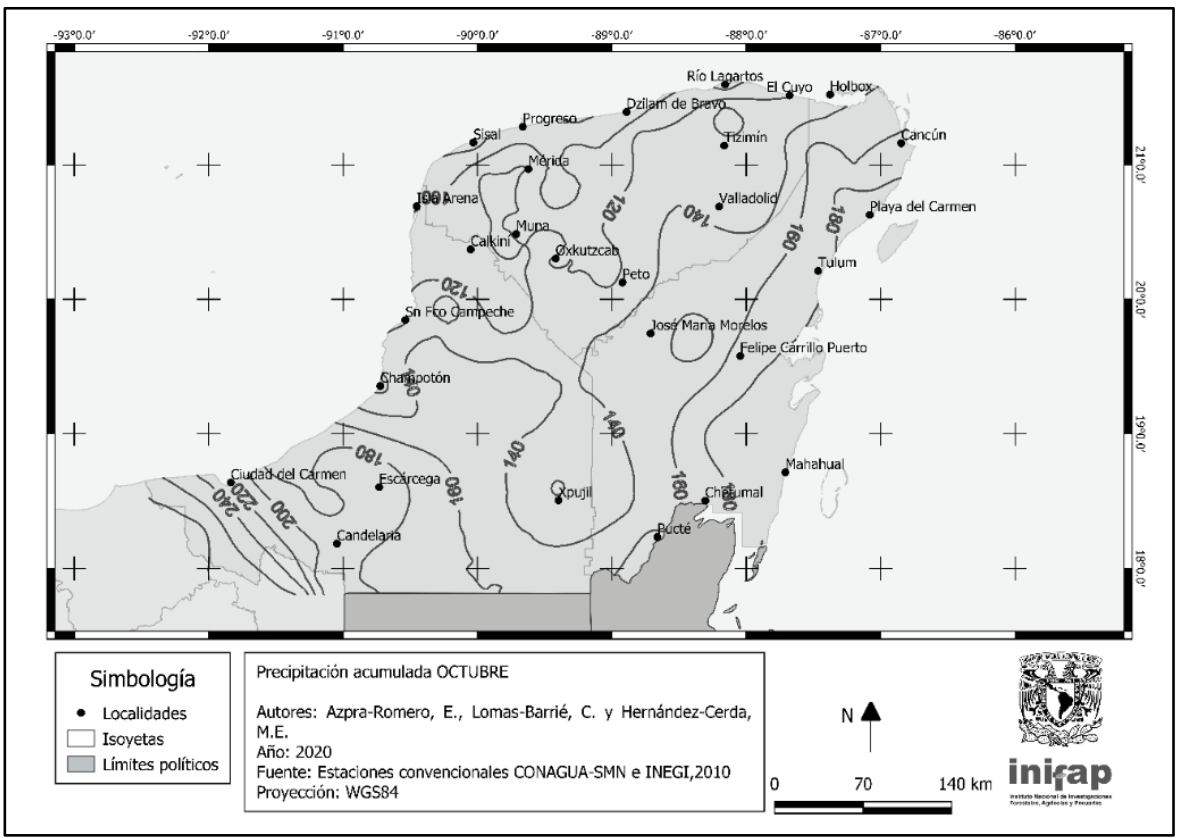

Figura 10. Precipitación media acumulada $(\mathrm{mm})$ del mes de octubre para la Península de Yucatán 
De un total de 87 estaciones analizadas del estado de Yucatán, se obtuvo que el $9.2 \%$ de éstas han registrado más de 20 veces las precipitaciones máximas en 24 horas; es decir, más de 100 mm por día. En Quintan Roo el 31.1\%, de un total de 61 estaciones. En Campeche el 18.9\% de un total de 80 estaciones utilizadas, de entre ellas, destaca la estación Palizada, qué registró 65 ocasiones precipitaciones máximas en 24 horas mayores a $100 \mathrm{~mm}$, ésta se localiza en el sureste del estado en donde se registran climas húmedos $(\mathrm{Am}(\mathrm{f}))$ y el resto están en la parte media en donde impactan una mayor cantidad de perturbaciones tropicales (Rosengaus et al., 2014 y Azpra et al., 2001).

\section{La TT Cristóbal}

La TT Cristóbal se formó a finales de mayo de 2020 a partir de la depresión tropical No. 3 (SMN, 2020b), en un área muy extensa de bajas presiones, de circulación ciclónica con convección embebida, en la zona que se le llama Giro Centroamericano (Papin et al., 2017), este sistema, aunque categorizado por su circulación (ciclónico o anticiclónica) en la alta troposfera en coincidencia con circulación ciclónica en superficie que ocasiona lluvias abundantes, debido a la convección profunda que presenta asociada a este vórtice, que se localiza en las cercanías de Centro América (Aldinger and Stapler,1998; American Meteorological Society 2016). Desde el 31 de mayo, de acuerdo con los modelos que se manejan en el Centro Nacional de Huracanes de Miami (NHC por sus siglas en inglés) se pronosticaba su fortalecimiento hasta alcanzar la categoría de TT (NOAA, 2020e); sin embargo, lo hizo hasta el 2 de junio para convertirse en la TT más prematura, con la letra “C” que afectaría a la Península de Yucatán (NHC-NOAA, 2020). A partir del 1 de junio y hasta el 5 junio, Cristóbal estuvo tres días en el sur del Golfo de México, entró a tierra por Campeche y posteriormente se desplazó hacia el norte para terminar impactando el estado de Luisiana, EU como TT cargada de humedad y con una importante marejada ciclónica (Figura 11). Los remanentes de la TT continuaron con su trayectoria hasta los Grandes Lagos, Canadá como lo indicaron los boletines de la NOAA (2020f) sin embargo, con el cambio de latitud la TT se convirtió en subtropical perdiendo interés en ella para este estudio.

A continuación, se muestra la distribución espacial diaria de la lluvia que dejó esta TT durante el tiempo que duró su recorrido por la Península de Yucatán. 
Fuente: Elaboración propia con datos del INEGI, 2010 y la trayectoria del Centro Nacional de Huracanes.

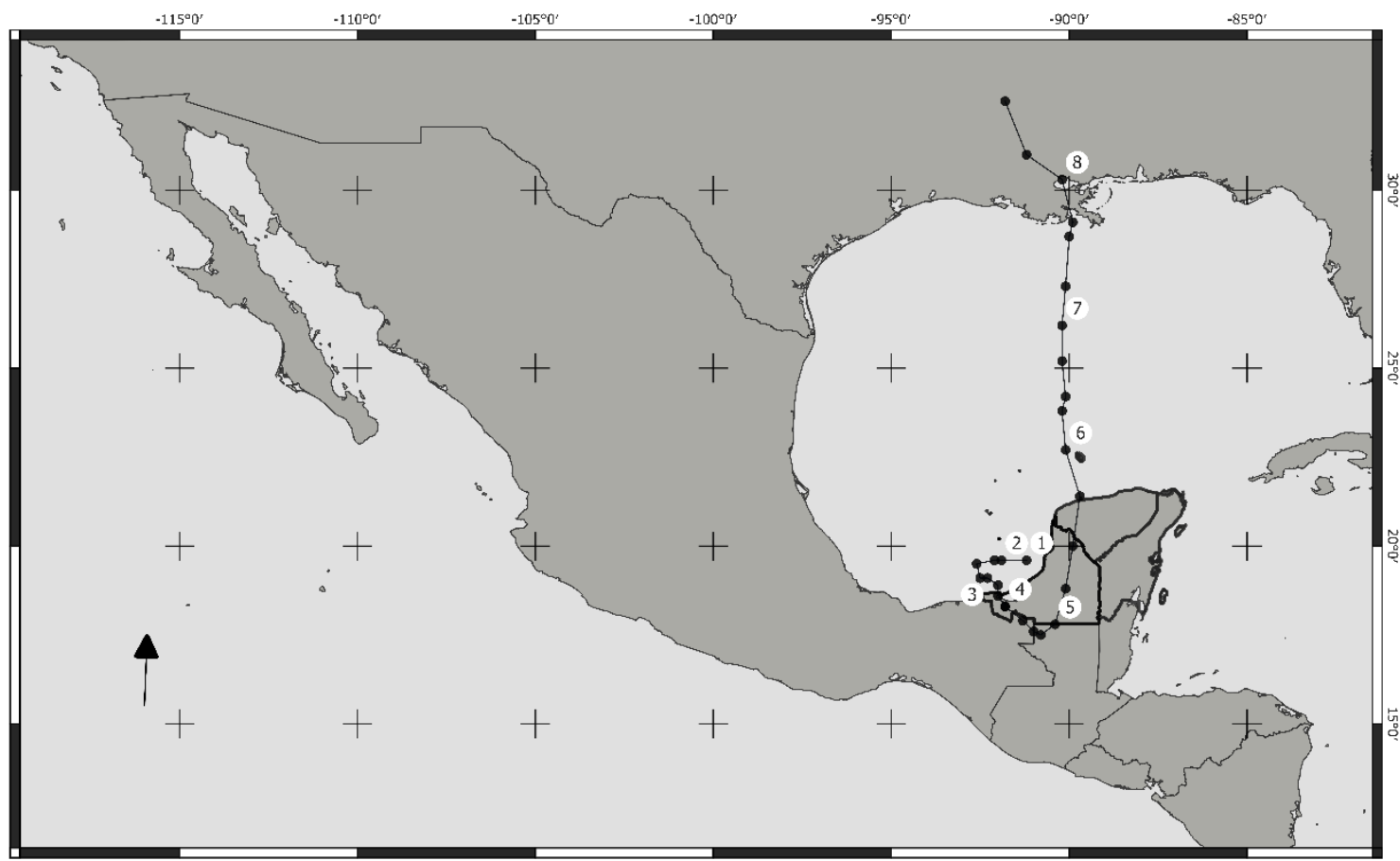

Figura 11. Trayectoria de la TT Cristóbal (01-08 junio, 2020). Los números indican los días del mes de junio.

El 31 de mayo (Figura 12), se observa un área con valores mayores a $40 \mathrm{~mm}$ en la parte centro y sur de la Península, alcanzado registros de $120 \mathrm{~mm}$ al sureste de Yucatán y norte de Quintana Roo y más de $100 \mathrm{~mm}$ al suroeste de Mérida. El máximo de lluvia en forma puntual que se registró en este día fue en la estación Cuzama del estado de Yucatán con 150 mm. Al día siguiente (1 de junio) su intensidad aumentó y estaba cerca de alcanzar la categoría de TT, su movimiento, era muy lento y la sucesión de crestas y valles del campo barométrico hacía suponer que haría meandros antes de que se moviera hacia el norte, con alguna posibilidad de adentrarse en el continente, cuya cercanía no favorece un incremento sustancial en la intensidad del ciclón.

El 1 de junio (Figura 13), el área de más de 40 mm registrada el día anterior, aumenta en extensión, ahora se extiende al norte de la Península, se observan dos máximos de lluvia, mayores de $80 \mathrm{~mm}$ al sur de la zona de estudio en los límites de los Estados de Campeche y Quintana Roo y el otro al norte en la parte centro del estado de Yucatán, entre Mérida y Valladolid, donde la cantidad de lluvia recibida fue mayor de 220 mm. La estación de Piste, en Yucatán con un registro de $225 \mathrm{~mm}$, fue el valor más alto de este 1 de junio. 
Fuente: Elaboración propia con datos de las estaciones convencionales CONAGUASMN, meteorológicas automatizadas de CONAGUA-SMN, INIFAP, UNAM e INEGI, 2010.

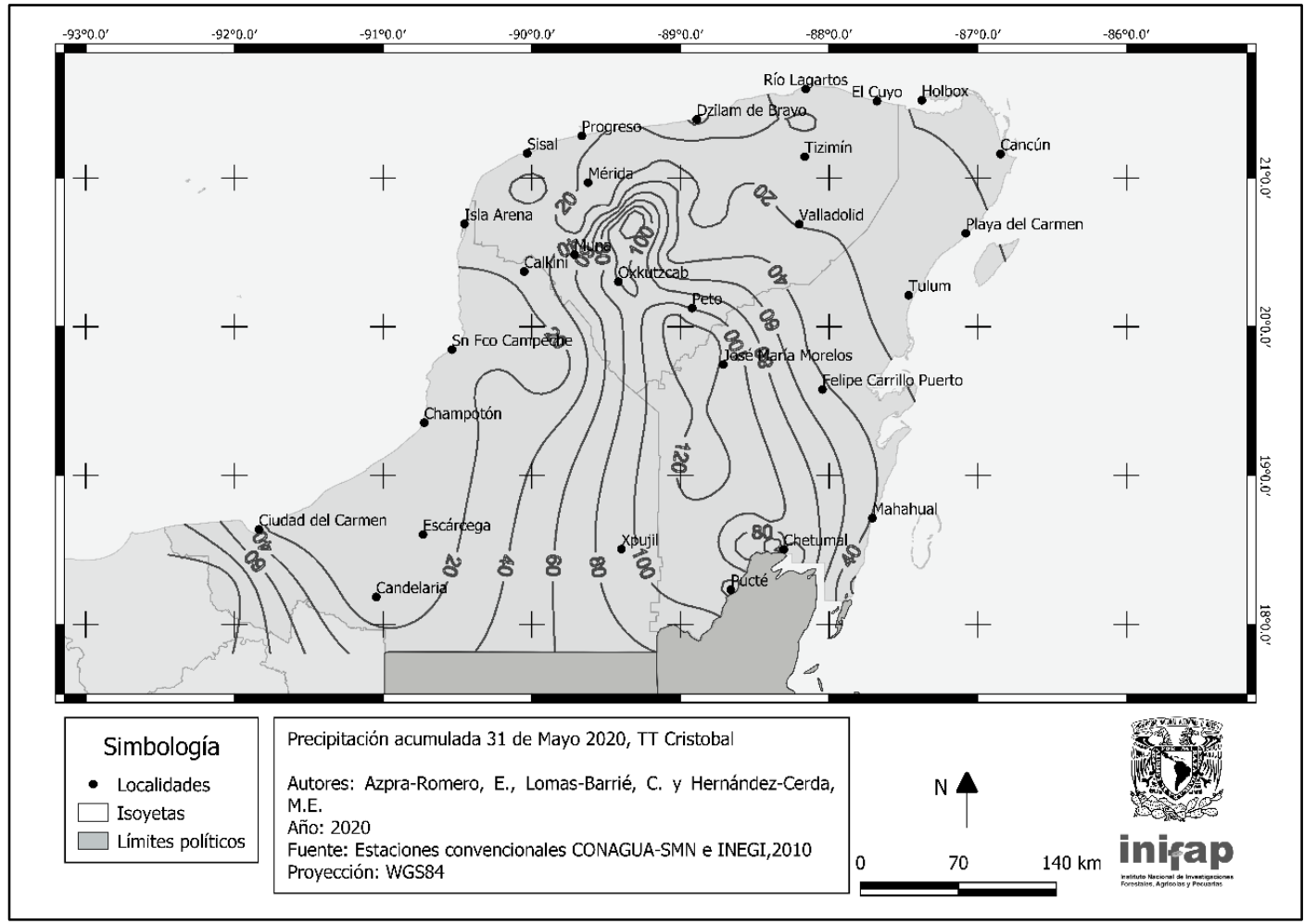

Figura 12. Precipitaciones (mm) del 31 de mayo de acuerdo a los datos de las estaciones convencionales de superficie, en la Península de Yucatán.

El 2 de junio (Figura 14), la lluvia aumenta tanto en intensidad como espacialmente. El área con más de $80 \mathrm{~mm}$ en este día abarca casi todo el estado de Yucatán y se extiende al norte de Campeche, dentro de esta área se registran dos máximos, uno al noreste con valores mayores a 160mm y más de 180 mm sobre el límite de estos dos Estados. La estación Bolonchen del estado de Campeche, registró el valor más alto, con un volumen de lluvia de $225 \mathrm{~mm}$.

Para el día 3 de junio (Figura 15), la organización de las bandas nubosas denotaba mayor altura de las cúspides de mayor desarrollo vertical en las cercanías del centro del sistema en concurrencia con vientos de alrededor de 50kt (del radar de Sabancuy), respaldaron su declaración como TT de nombre Cristóbal. Su movimiento se dirigió hacia el sureste, pero erráticamente, esto ocasionado por un anticiclón que se localizaba sobre el Golfo de México. Su afectación sobre la Península de Yucatán es clara ya que se encuentra muy cercano a las costas de Campeche. 
Fuente: Elaboración propia con datos de las estaciones convencionales CONAGUASMN, meteorológicas automatizadas de CONAGUA-SMN, INIFAP, UNAM e INEGI, 2010.

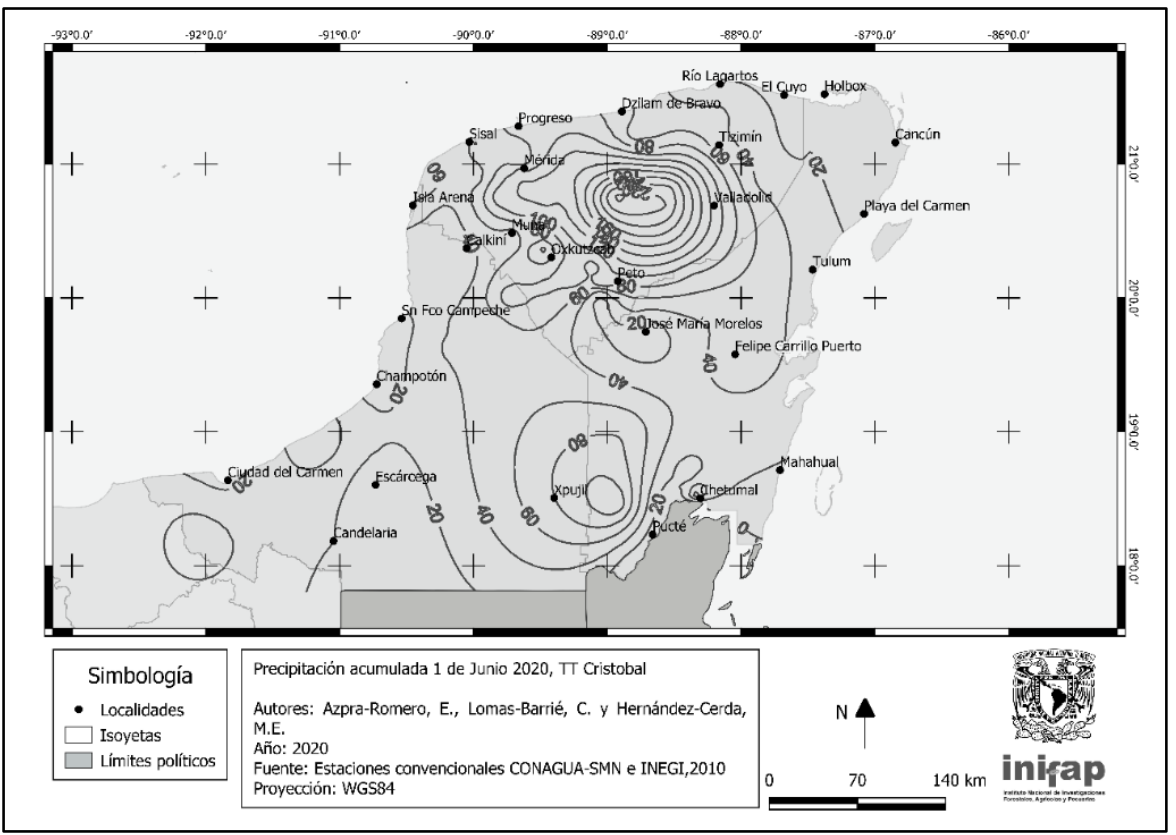

Figura 13. Precipitaciones $(\mathrm{mm})$ del 1 de junio de acuerdo a los datos de las estaciones convencionales de superficie, en la Península de Yucatán

Fuente: Elaboración propia con datos de las estaciones convencionales CONAGUASMN, meteorológicas automatizadas de CONAGUA-SMN, INIFAP, UNAM e INEGI, 2010.

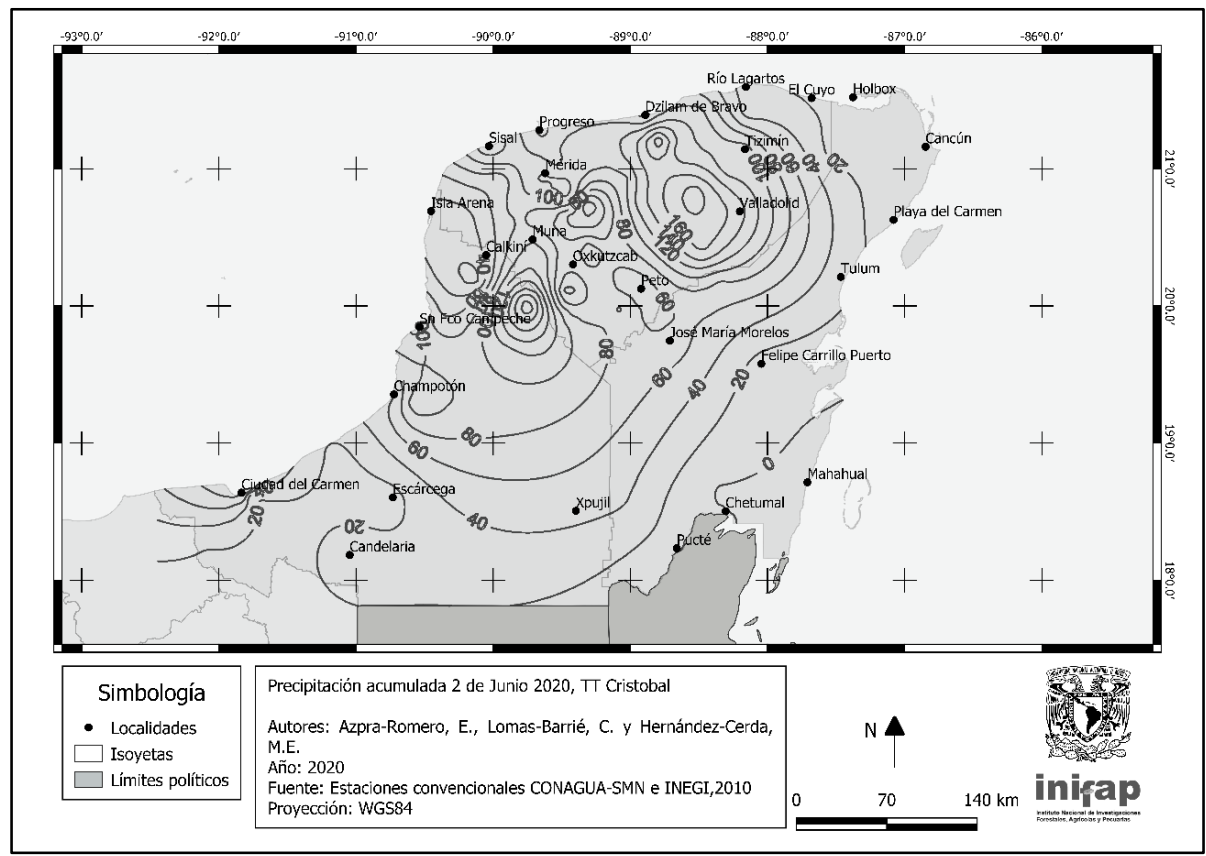

Figura 14. Precipitaciones $(\mathrm{mm})$ del 2 de junio de acuerdo a los datos de las estaciones convencionales de superficie, en la Península de Yucatán 
En este día, la lluvia sigue en aumento, el área de más de $80 \mathrm{~mm}$ abarca casi toda la Península. Los registros son mayores de $120 \mathrm{~mm}$ en casi todo el suroeste y en un área al norte del estado de Campeche. En la parte central del estado de Yucatán entre Mérida y Valladolid la lluvia es mayor, alcanzando valores de más de $200 \mathrm{~mm}$. Puntualmente el valor de lluvia más alto fue el que registró la estación Holca, en el estado de Yucatán con 340 mm.

Fuente: Elaboración propia con datos de las estaciones convencionales CONAGUASMN, meteorológicas automatizadas de CONAGUA-SMN, INIFAP, UNAM e INEGI, 2010.

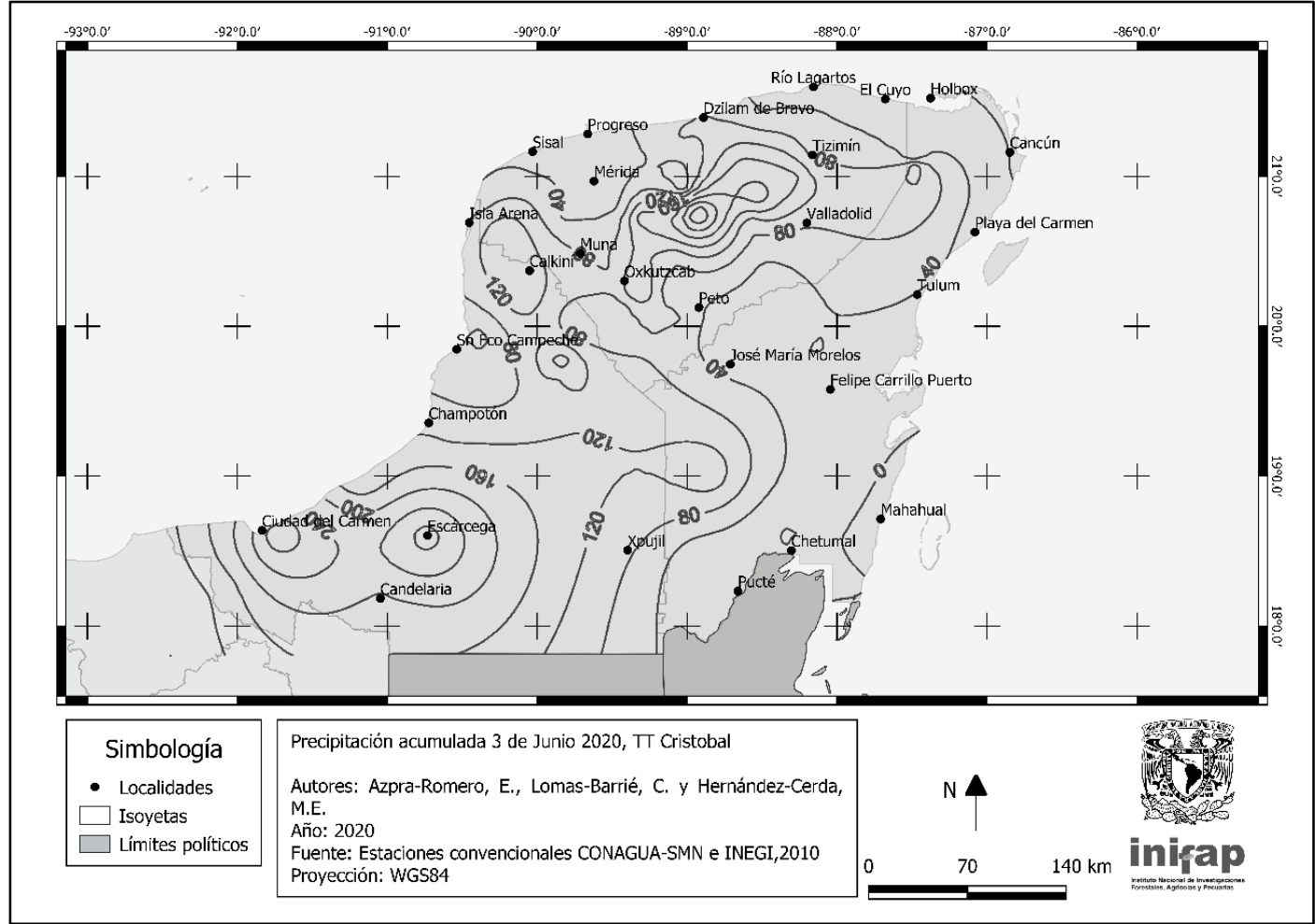

Figura 15. Precipitaciones $(\mathrm{mm})$ del 3 de junio de acuerdo a los datos de las estaciones convencionales de superficie, en la Península de Yucatán

El día cuatro la TT Cristóbal (Figura 16), se localiza sobre tierra en el estado de Campeche y alcanza su punto más meridional sobre Guatemala. En este día se registran los valores más altos de precipitación. El área de más de $80 \mathrm{~mm}$ de lluvia se reduce de extensión, y se localizan dos áreas, una al sureste de Campeche donde alcanza valores de más de $120 \mathrm{~mm}$ y la otra que abarca toda la superficie de la entidad de Yucatán, donde los registros alcanzan valores mayores a $260 \mathrm{~mm}$ al suroeste del estado y más de $200 \mathrm{~mm}$ al noroeste de Valladolid. Es en este día cuando se registran 
360 mm en la estación Tekax, en el centro de Yucatán, que es la cantidad más alta de lluvia de estos 6 días analizados y de todo el periodo de registro de esta estación.

Fuente: Elaboración propia con datos de las estaciones convencionales CONAGUASMN, meteorológicas automatizadas de CONAGUA-SMN, INIFAP, UNAM e INEGI, 2010.

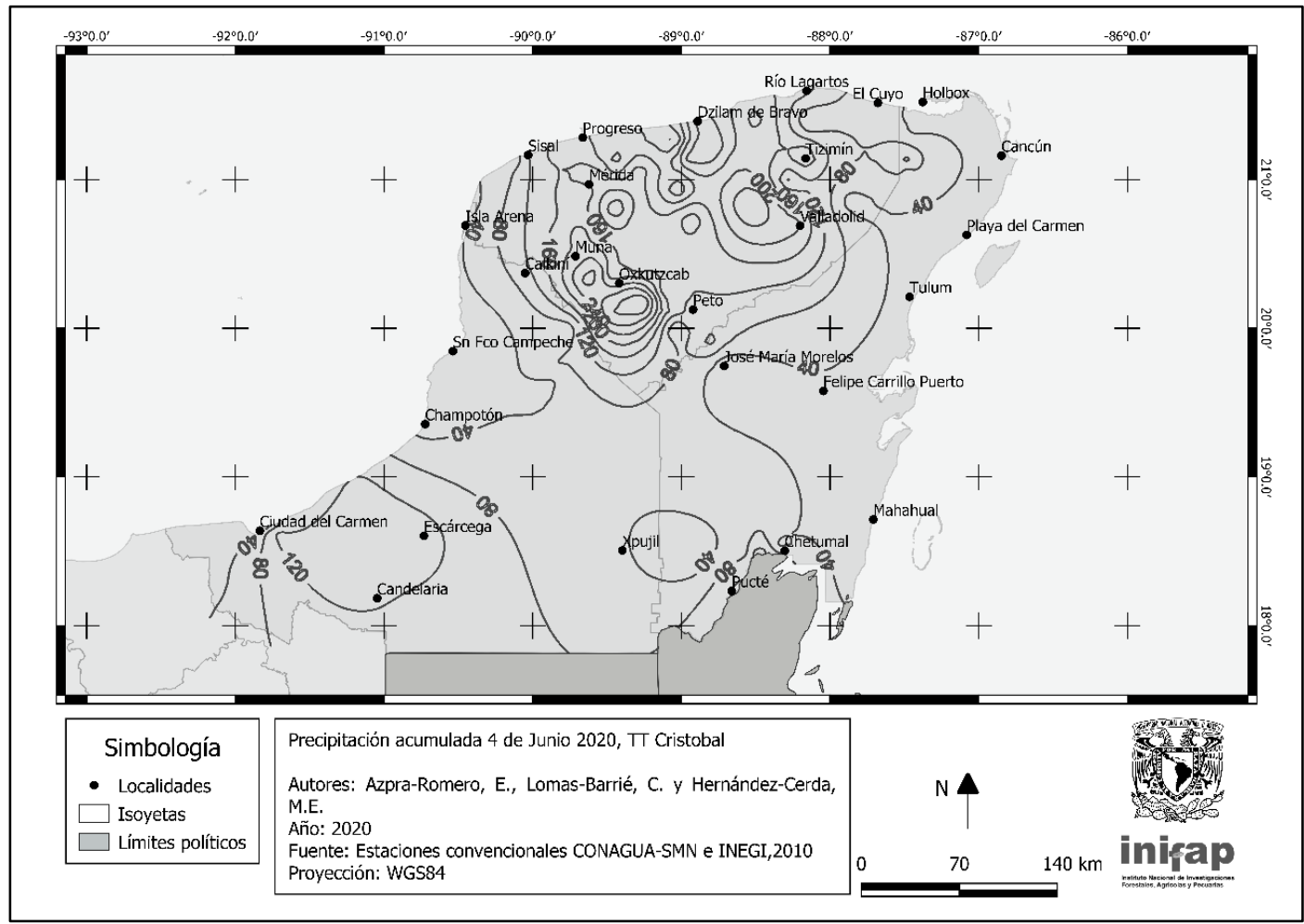

Figura 16. Precipitaciones $(\mathrm{mm})$ del 4 de junio de acuerdo a los datos de las estaciones convencionales de superficie, en la Península de Yucatán

La TT Cristóbal, posteriormente se comienza a mover hacia el norte con una componente oriental hasta salir del continente por las costas de Yucatán antes de que empiece el día 6, durante este tiempo, el movimiento hacia el este por parte del anticiclón de las Azores influyó en este desplazamiento fuera hacia el norte.

En el comportamiento de la lluvia en la península, el día 5 de junio (Figura 17), se puede observar una disminución del área con más de $80 \mathrm{~mm}$ que abarca el oeste del estado de Yucatán y la mitad norte del de Campeche, donde aumentan los valores a más de $140 \mathrm{~mm}$ en el límite interestatal y más de 100 mm al sur de la Península sobre los límites de Campeche con Quintana Roo. Dos áreas, pero de menor tamaño que registran más de $80 \mathrm{~mm}$ se localizan una al este de Mérida y la otra al noroeste de la Península, en las áreas circunvecinas de Playa del Carmen, Quintana Roo, donde los 
registros de lluvia son mayores de $110 \mathrm{~mm}$. La estación con el máximo de lluvia de este día fue el registrado nuevamente en la estación Bolonchen, Campeche con 204 mm.

Fuente: Elaboración propia con datos de las estaciones convencionales CONAGUASMN, meteorológicas automatizadas de CONAGUA-SMN, INIFAP, UNAM e INEGI, 2010.

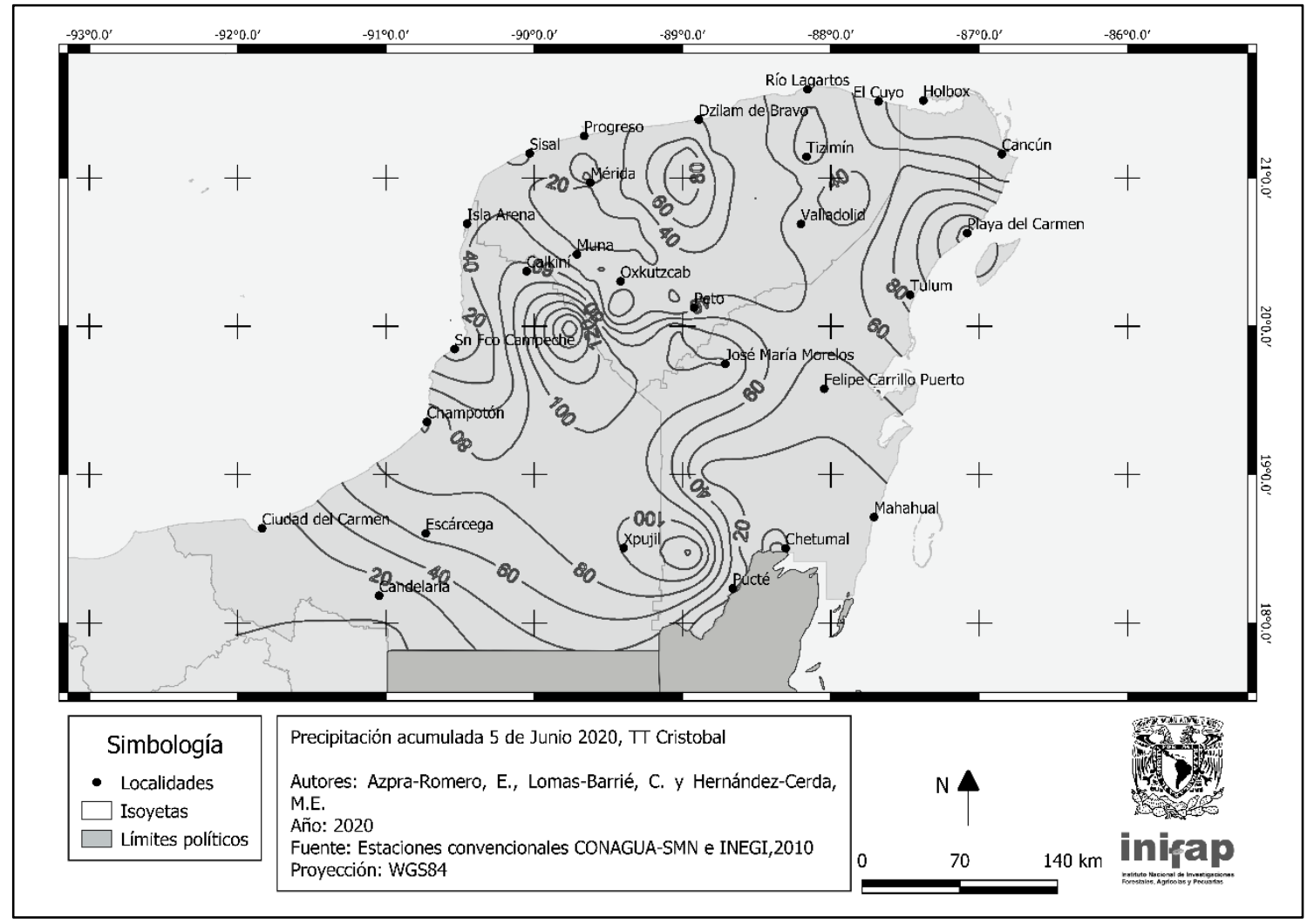

Figura 17. Precipitaciones $(\mathrm{mm})$ del 5 de junio de acuerdo a los datos de las estaciones convencionales de superficie, en la Península de Yucatán

En la imagen de satélite del GOES en una composición del visible (Figura 18) se observa el área de afectación sobre la Península de Yucatán el día 5 de junio.

La lluvia acumulada de estos seis días (Figura 19), se observa que se recibieron más de 300 mm en la mayor parte de la Península de Yucatán, siendo los mayores registros en el estado de Yucatán, en dos áreas una con valores de más de 600 mm al suroeste de Yucatán sobre el límite estatal con Campeche y al oeste de la localidad Peto; la otra área localizada entre Mérida y Valladolid, en donde se registraron lluvias mayores de $850 \mathrm{~mm}$. Es en esta región es donde se localizan las estaciones Dzitás y Holca, Yuc. que fueron las que tuvieron las mayores precipitaciones acumuladas, 959 mm y 905.6 mm respectivamente. La estación Holca fue la que también registró el máximo de $360 \mathrm{~mm}$ en un día. 
Fuente: (GOES, 2020)

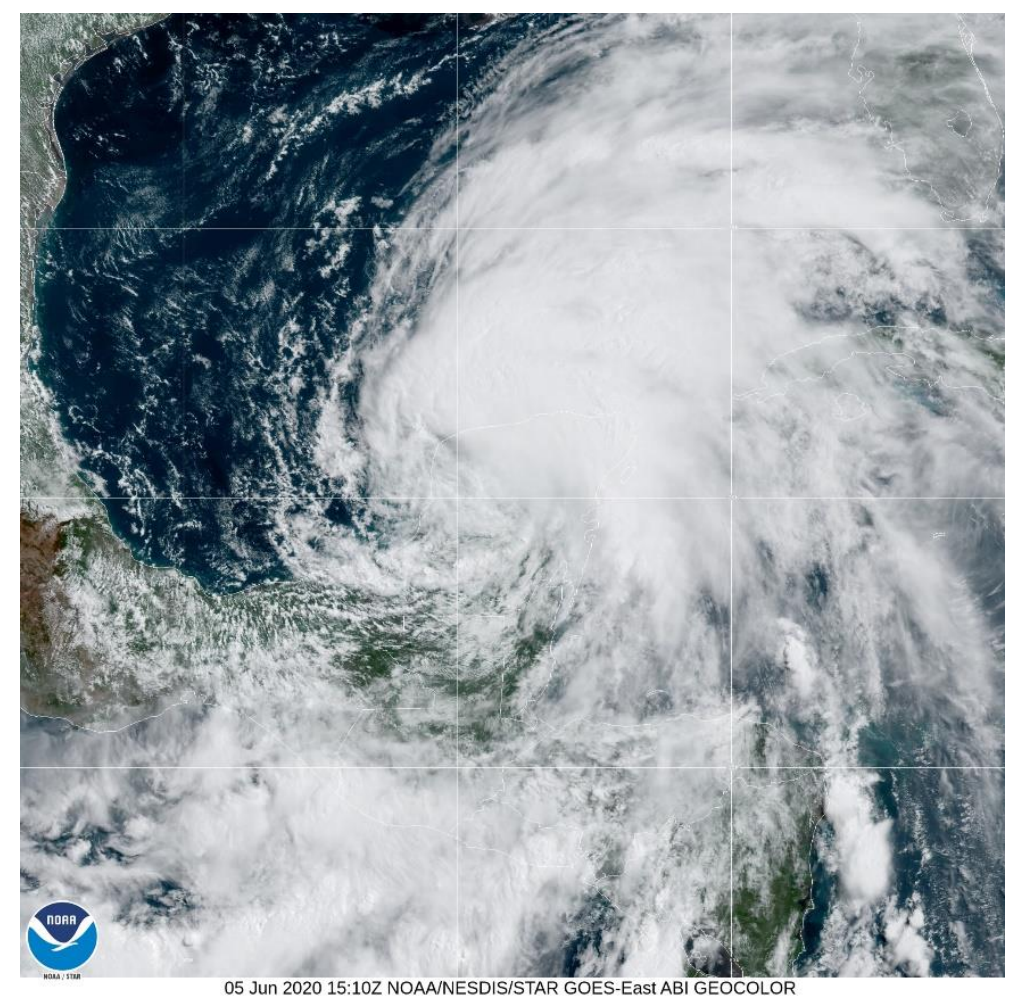

Figura 18. Imagen del satélite GOES 16 composición ABI GEOCOLOR del 5 de junio a las 15:10UTC.

\section{Condiciones climáticas de los CT vs TT Cristóbal}

Las precipitaciones en los Estados que constituyen la Península de Yucatán desde el 31 de mayo hasta el 5 de junio alcanzaron valores por arriba de la media mensual en algunos lugares, aun cuando la intensidad del CT estaba disminuyendo. Esto se puede apreciar en la gráfica de la Figura 20, que muestra los valores de lluvia diarios más altos, los acumulados en los seis días y la media anual y mensual. Los valores que se observan sobre las barras de la suma de la lluvia diaria indican el porcentaje que se alcanzó respecto a la media anual y el que se localiza sobre la barra de los registros de la máxima diaria se calculó respecto a la media mensual. En esta gráfica se puede observar que las máximas diarias fueron superiores al $100 \%$ con respecto del valor mensual correspondiente. En el caso de la estación Cuzama, Yucatán, recibió $150 \mathrm{~mm}$ el 31 de mayo lo que representa el $240 \%$ de la precipitación registrada para ese mes, en el cual inicia la temporada de lluvias de la mitad caliente del año. Con relación a la lluvia acumulada en los seis días de la TT, en la gráfica se puede apreciar que en la estación Holca, Yucatán, llovió el 94\% de lo que se recibe en el año. 
Fuente: Elaboración propia con datos de las estaciones convencionales CONAGUASMN, meteorológicas automatizadas de CONAGUA-SMN, INIFAP, UNAM e INEGI, 2010 y la trayectoria del Centro Nacional de Huracanes.

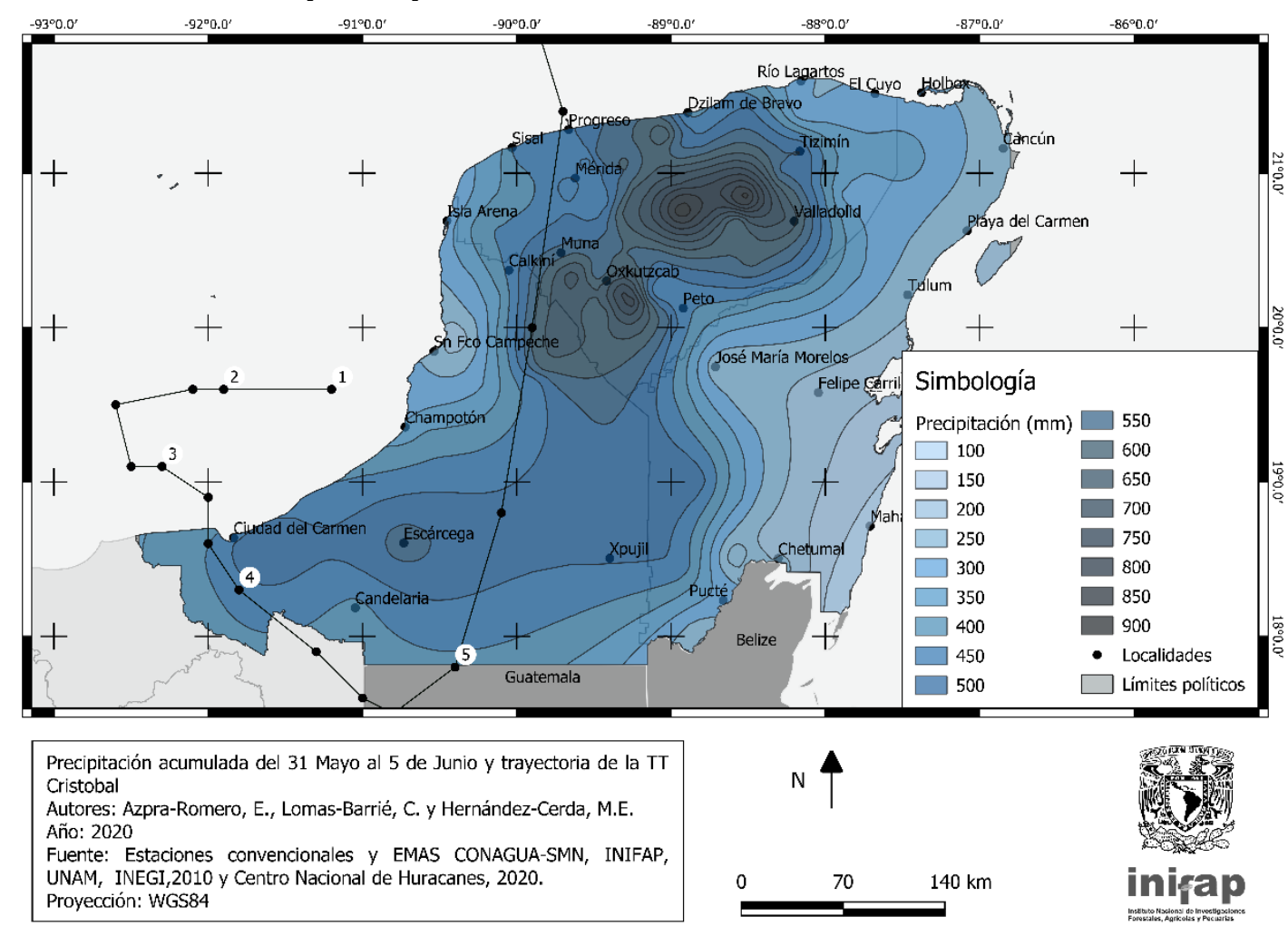

Figura 19. Precipitaciones acumuladas (mm) del 31 de mayo al 5 de junio de 2020, registradas en las estaciones convencionales y automáticas

Se obtuvo la frecuencia del paso de los CT por la Península, siendo un total de 77 perturbaciones tropicales que entraron durante el periodo de 1960-2019 (Tabla 1), 36 alcanzaron la categoría de TT según el NHC-NOAA. Quintana Roo es el estado más afectado por la entrada de estos sistemas meteorológicos, por su posición en la costa del Mar Caribe. Sin embargo, de la comparación de los valores de este cuadro con las áreas de precipitación tanto anuales, semestrales y mensuales, no se puede establecer que una mayor afectación por CT produzca una mayor cantidad de precipitación. Es decir, los CT aunque sean productores de una gran cantidad de lluvia por evento, no producen gran impacto en la escala climatológica. 
Fuente: Elaboración propia con datos de las las estaciones convencionales CONAGUASMN, meteorológicas automatizadas de CONAGUA-SMN, INIFAP, UNAM

Precipitaciones máximas

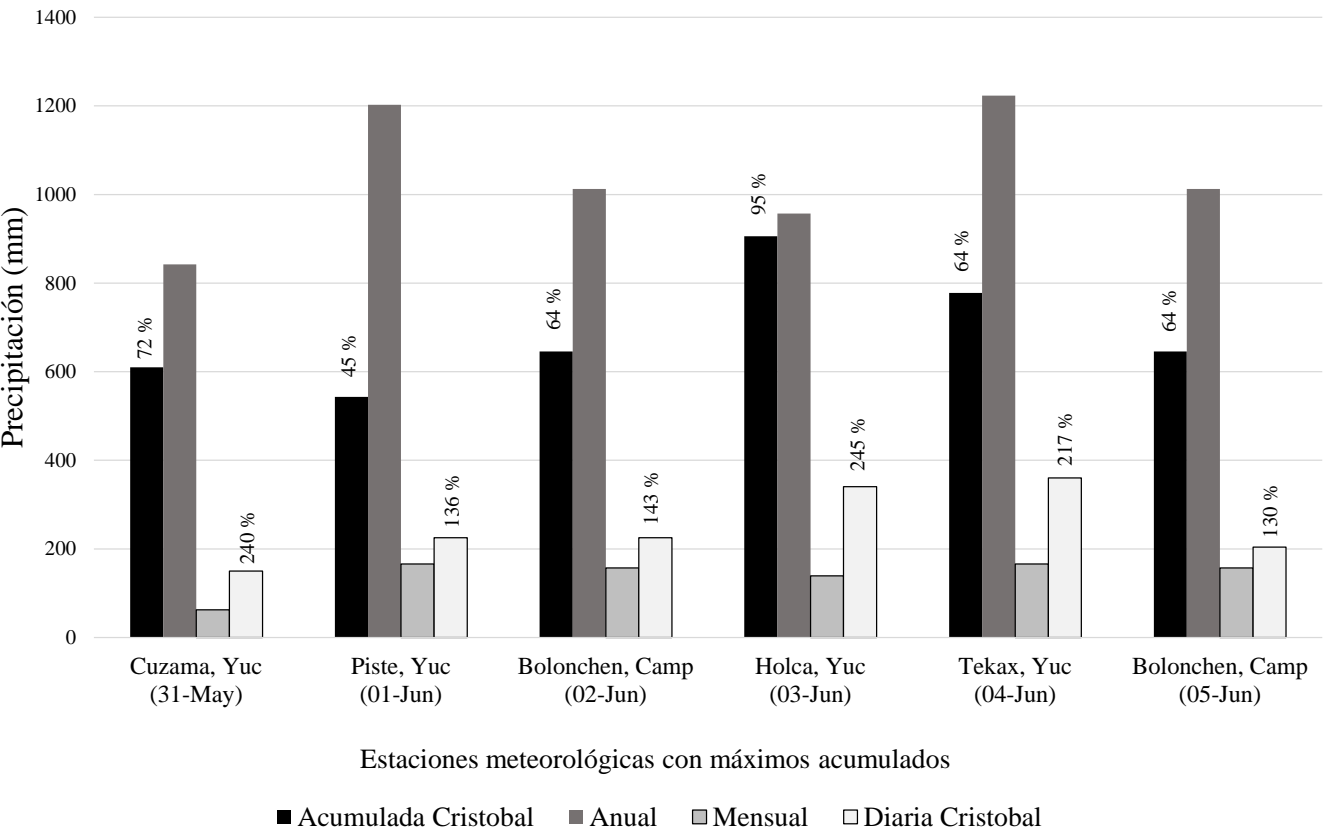

Figura 20. Precipitación (mm) acumulada de los seis días de Cristóbal, la media anual, la media mensual de junio y la máxima diaria durante el paso de la TT Cristóbal en la Península de Yucatán. Los porcentajes representan el acumulado por Cristóbal respecto a la media anual y la lluvia diaria acumulada durante Cristóbal respecto a la media mensual de junio.

Tabla 1. Meteoros que entraron a la Península de Yucatán, durante el periodo de 1960 a

\begin{tabular}{|l|c|c|c|c|c|c|c|c|}
\hline Entidad & DT & TT & H1 & H2 & H3 & H4 & H5 & TOTAL \\
\hline Campeche & 6 & 10 & 1 & 1 & 1 & & & 19 \\
\hline Q. Roo & 10 & 16 & & 1 & 1 & 3 & 2 & 33 \\
\hline Yucatán & 10 & 10 & & 2 & 1 & 1 & 1 & 25 \\
\hline Total & 26 & 36 & 1 & 4 & 3 & 4 & 3 & 77 \\
\hline
\end{tabular}

Depresión tropical (DT), Tormenta tropical (TT), Huracán (H) el numeral está basado en las categorías de la escala de Saffir-Simpson (NOAA, 2009).

Fuente: Elaboración propia con datos de la NOAA.

\section{Impactos de la TT Cristóbal}

La trayectoria de Cristóbal fue anómala desde su formación hasta su desplazamiento y debilitamiento. Cristóbal afectó a la Península de Yucatán entre los días 1 al 6 de junio, con efectos 
de TT desde Coatzacoalcos, Veracruz hasta Campeche y con temporal de lluvias sobre los Estados de Yucatán y Quintana Roo. Sus efectos se sintieron en los Estados vecinos, de Tabasco, Chiapas y Veracruz. Las lluvias provocadas por Cristóbal hicieron que las autoridades tuvieran que evacuar personas ya que ocasionó deslizamientos de tierras en Chiapas y Veracruz así cómo inundaciones en carreteras de Chiapas, Campeche, Tabasco y Quintana Roo (El Financiero, 2020; Briceño, 2020). Existen diferentes estimaciones de las afectaciones según la fuente que se consulte, entre ellas se encuentran: en Campeche 719 personas fueron desalojadas (Acosta, 2020) En Yucatán, 8173 viviendas sufrieron daños (Luces del Siglo, 2020) y en Quintana Roo 3500 familias fueron afectadas (Puerto, 2020a). En el transcurso del 3 de junio, la TT Cristóbal tocó tierra a 20 kilómetros de Ciudad del Carmen, Campeche y fue moviéndose por el sureste del país hasta llegar a Yucatán y Quintana Roo provocando diversos daños como los que se lograron documentar aún días después de pasado el meteoro (Figura 21).

Fuente: Elaboración propia, fotografías tomadas por Lomas-Barrié, C.T. 2020.

a)
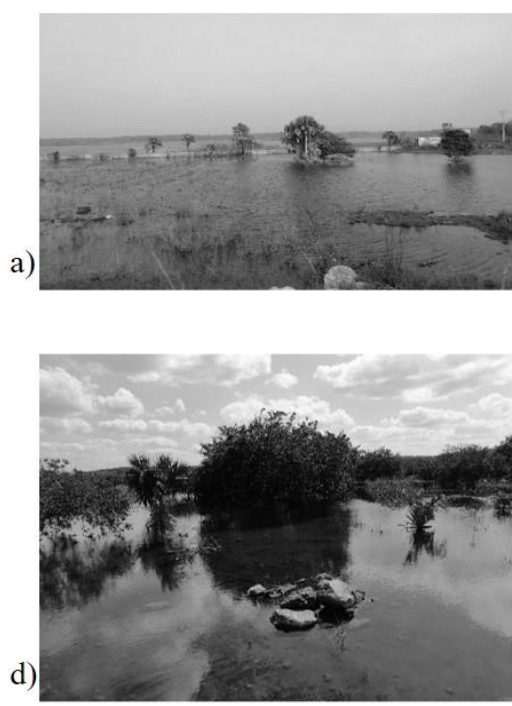
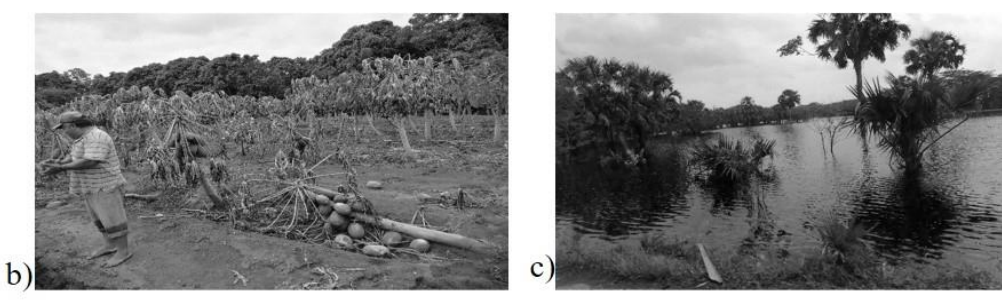

e)
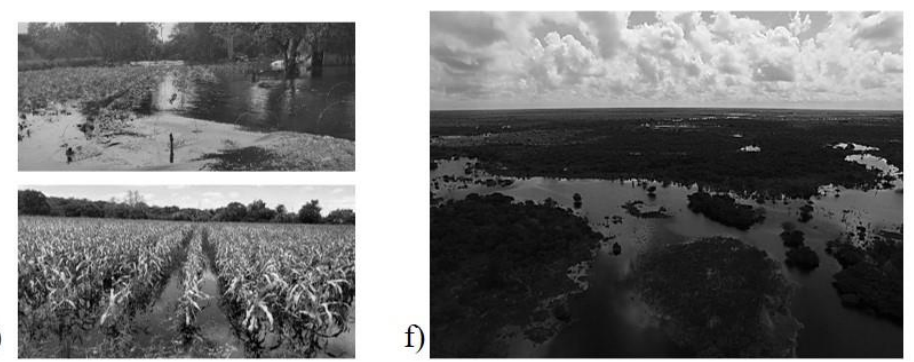

Figura 21. Efectos de las lluvias severas e inundaciones pluviales a) Zona agrícola de hortalizas y Maíz en Tenabo, Camp. 10 de junio, b) Perdida total de cultivo de papaya en Tikinmul, Camp. 10 de junio, c) Zona agrícola de Maíz y hortalizas en José María Morelos (JMM), Quintana Roo 24 de junio, d) Cultivo de limón en El Verdón JMM, Quintana Roo 09 de junio, e) Cultivos de Maíz y Calabaza en Muna, Yuc 11 de junio, y f) Pastizales Río Lagartos, Yuc. 12 de junio. 


\section{Discusión}

Aun cuando la precipitación asociada a los CT significa eventos con altos volúmenes de precipitación, en la climatología de la lluvia no se aprecia una influencia en el promedio de largo plazo para la Península de Yucatán, ya que las áreas de impacto de los CT, en los mapas mensuales, semestrales y anual no es posible identificar la moda en la trayectoria espacial que siguen estos fenómenos respecto a las precipitaciones acumuladas registradas en las estaciones. Es decir, la mayoría de las trayectorias se delinean de este a oeste cruzando la península (Rosengaus et al., 2014 y 2016); mientras que la mayor cantidad de lluvia se localiza en las vecindades de los estados de Tabasco y Chiapas, hacia el suroeste. Esta característica es clara tanto en la lluvia anual como en la correspondiente a la mitad caliente del año (ver Figuras 3 y 4).

A pesar de que este CT no alcanzó la categoría de huracán, por la velocidad de sus vientos, a su lento desplazamiento y el tiempo de permanencia en tierra, provocó lluvias torrenciales, esto es entre 150 a $250 \mathrm{~mm}$ en 24 horas (Secretaría de Marina, 2020) a lo largo de 6 días en las tres entidades que conforman la Península de Yucatán. La distribución espacial de este acumulado se muestra en el mapa que tiene señalada la trayectoria de la TT Cristóbal (Figura 19) y el porcentaje de área para cada uno de los intervalos de lluvia se muestran en la (Figura 22). Durante estos 6 días las áreas que recibieron lluvias extraordinarias (secretaria de Marina, 2020) entre 250 a $500 \mathrm{~mm}$ fueron del 76\% de la superficie de la Península, de donde el intervalo entre 450 y $500 \mathrm{~mm}$ fue el que abarcó la mayor superficie (37\%).

Fuente: Elaboración propia

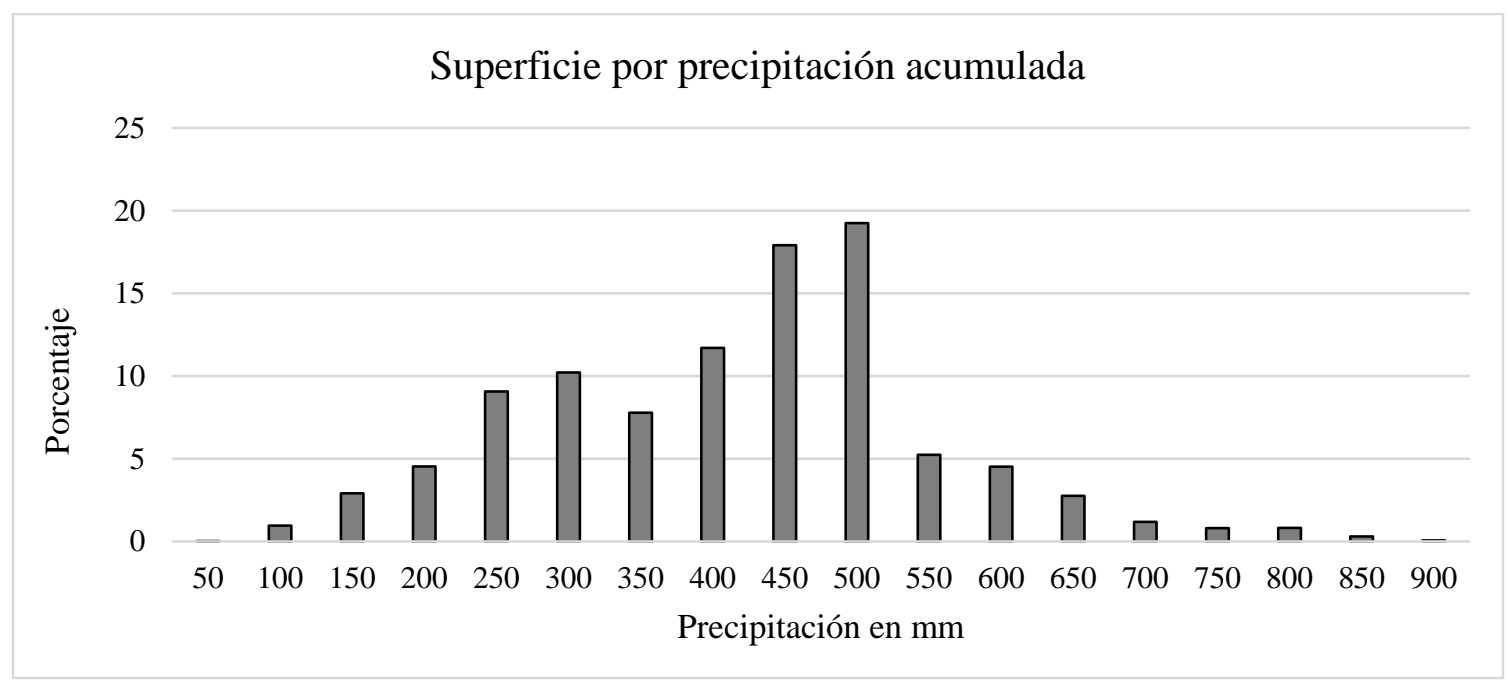

Figura 22. Superficie de la Península de Yucatán por rango de precipitación acumulada durante el periodo de Cristóbal. 
La trayectoria de la TT Cristóbal no se aproxima a la típica parábola que describen los CT, se movió inicialmente hacia el sureste cruzando Yucatán, hasta alcanzar el Golfo de México y para continuar moviéndose hacia el norte y, eventualmente desplazarse hasta los Grandes Lagos. Este comportamiento se debe a que su movimiento estuvo influido por un sistema meteorológico migratorio propio de la transición entre las épocas fría y caliente del año como lo menciona Azpra et al. (2001).

Con base en el análisis espacial y cuantitativo de los registros de la lluvia de las estaciones que se hizo, se pudo establecer que para que un CT afecte, no es necesario que sea intenso ya que la velocidad de traslación es un factor importante en la magnitud de los daños que ocasiona, sino por algunos factores relacionados con la vulnerabilidad (RosengausMoshinsky y Sánchez-Sesma, 1990). Por ejemplo, el CT Isidore fue un sistema de mayor intensidad que tenía un tiempo de vida que le permitió madurar lo suficiente desde su origen en las costas norte de Venezuela y, al atravesar el Caribe, desarrollarse hasta alcanzar la categoría tres en la escala Saffir-Simpson, intensidad con la que penetró por el norte de Yucatán el 21 y 22 de septiembre de 2002 (NOAA-NHC, 2002). Este sistema a pesar de tener intensidad de huracán mayor, se movió rápidamente hacia el norte sin producir cantidades de lluvia similares a Cristóbal, sin embargo, Isidore solo produjo precipitaciones máximas el 21 y 22 septiembre de 2002 de 265.7 y $300 \mathrm{~mm}$ respectivamente. El promedio diario más alto fue de 98.5 mm el día 22 septiembre de 2002 y Cristóbal dejó 117.3 mm en promedio el día 4 de junio de 2020.

En el caso del CT Gilbert en 1988 representa otro ejemplo claro de que la mayor intensidad no necesariamente está relacionado con una mayor cantidad de precipitación, ya que su rápido paso del mar Caribe al Golfo de México sucedió en menos de 2 días, las precipitaciones aunque importantes, se dieron el 14 de septiembre, la precipitación promedio fue de $90.6 \mathrm{~mm}$, (aún se encontraba en el Caribe, frente a Cozumel) y disminuyeron rápidamente los días siguientes, el 16 de septiembre se halló a mitad del Golfo de México (NHC, 1988).

En contraste, Cristóbal fue un sistema que se originó al principio de la temporada, cuando no se esperaba que alcanzara su máximo potencial (lo que sucedió); sin embargo, su origen 
cercano a la península de Yucatán y su anómala trayectoria, contribuyeron a que su afectación, en lo que se refiere a precipitación, fuera más fuerte que sistemas más intensos.

Para tratar de explicar una de las causas que provocaron inundaciones y que, en algunos lugares como Hopelchén, permanecieron por más de 3 meses inundados (Puerto, 2020b; Por esto, 2020 a,b), se calculó la cantidad de lluvia acumulada en seis de las 90 estaciones de la red estudiada, la cual fue de 33,629 mm lo que equivale a una capa de más de 33 metros de altura sobre la superficie del suelo, que representa el $40 \%$ de lo que se recibe en el año. El estado de Yucatán recibió el 70\% de esa cantidad, que, al comparar con los mapas de las precipitaciones acumuladas normales, en 6 días llovió lo que se acumularía en los meses de junio, julio y agosto.

Los daños más importantes se dieron en el sector agropecuario, principalmente en cultivos agrícolas y en colmenas de abejas (Figura 21). También hubo efectos en viviendas inundadas, perdida de techos por el efecto del viento, daños en la infraestructura de las vías de comunicación, ocasionando grandes pérdidas económicas en los 3 Estados. El gobierno de Yucatán estimó las pérdidas en 5422 millones de pesos, de los cuales, 2066 millones de pesos corresponden al sector agrícola, 1389 millones al sector vivienda y 1968 millones al sector infraestructura, de acuerdo a la Secretaria de Seguridad y Protección Ciudadana encargada de las declaraciones de emergencia o desastre natural nacional, determinó y publicó en el Diario Oficial de la Federación del 12 al 19 de junio, diferentes categorías de emergencia para 11 municipios de Campeche, 4 de Quintana Roo y 81 de Yucatán (DOF, 2020 a,b,c) que dio lugar a solicitar ayuda al Fondo de Desastres Naturales (FONDEN), instancia gubernamental para apoyar a los municipios más afectados. De acuerdo a declaratorias en los diarios se estimó en 40 millones de pesos en Campeche (Tribuna Campeche, 2020), 4 mil millones de pesos en Yucatán (Diario de México, 2020) y 1 millón de pesos en Quintana Roo (Palco Noticias Zona maya, 2020).

\section{Conclusiones}

En la cartografía correspondiente a la precipitación anual acumulada, y de la mitad caliente del año, las áreas con mayores precipitaciones no coinciden con las de mayores altitudes, es decir, no se muestra una influencia orográfica en la distribución, no así con la distancia a las costas, sobre todo en la parte nor-oriental de la Península. 
No se observó una relación marcada entre la precipitación producida por los CT y la climatología de la precipitación anual y estacional, sin embargo, la intensidad de las precipitaciones durante un CT son una amenaza importante para la población y sus actividades económicas.

La TT Cristóbal es un ejemplo de que la afectación producida por un CT no necesariamente está relacionada con su intensidad, ya que este sistema produjo récords de precipitación en varias estaciones a pesar de no haber alcanzado la categoría de huracán. Asimismo, las inundaciones producidas fueron similares o mayores a sistemas de mayor intensidad tales como Isidore (2002) o Gilberto (1988).

La TT Cristóbal durante su recorrido por la Península de Yucatán causó pérdidas económicas en el sector primario y daños en la infraestructura urbana. Así mismo, aunque no fue posible evaluarlo en este estudio, al haberse dado durante la época de la pandemia por Covid-19, otro de sus efectos de la TT pudo ser el incremento en los contagios por este virus.

\section{Agradecimientos}

Met. Enrique Árcega por proporcionar información meteorológica, a los Ing. Sergio Peña Fierro y Juan Enrique Araiza Rodríguez del Centro Hidrometeorológico Regional "Mérida" de la CONAGUA, por los datos de las estaciones convencionales de la región.

\section{Referencias bibliográficas}

Acosta, P. M. (2020). Desalojos y daños en carreteras deja "Cristóbal" en Campeche. Diario de Yucatán. https://bit.ly/3a5OXQQ.

Aldinger, W. T., y Stapler, W. (1998). 1998 annual tropical cyclone report. Tech. Rep. ADA381675, JTWC, U.S. Naval Pacific Meteorology and Oceanography Center, B, 224 pp. https://bit.ly/3sdj7I6.

Álvarez Rodríguez, J. (2011). Estimación de la distribución espacial de la precipitación en zonas montañosas mediante métodos geoestadísticos (Tesis doctoral). E.T.S.I. Caminos, Canales y Puertos (UPM). http://oa.upm.es/8820/

Álvarez Rodríguez, J., Llasat, M.C., y Estrela T. (2019). Development of a hybrid model to interpolate monthly precipitation maps incorporating the orographic influence. International Journal of Climatology. 1-14. https://doi.org/10.1002/joc.6051 
American Meteorological Society (2016). Glossary of Meteorology. https://bit.ly/3o4cbMU

Azpra, E., Carrasco, A. G., Delgado, D. O., y Villicaña, C. F. J. (2001). Los ciclones tropicales de México. 1.6.1. Temas selectos de Geografía de México. Instituto de Geografía, UNAM. 127-120.

Briceño, M. E. (2020). Más de mil personas damnificadas por "Cristóbal en Campeche". La Jornada. https://bit.ly/3g546p7.

Diario de México. (2020). Tormenta tropical "Cristóbal" dañó 95mil hectáreas de cultivos en Yucatán: gobernador. Diario de México. https://bit.ly/3uNyYim

Díaz Castro, S. C (2010). Variabilidad de los ciclones tropicales que afectan a México. Interciencia, 35(4), 306-310.

Diario Oficial de la Federación. Declaratoria de Emergencia. (2020a). DOF:15/06/2020. Yucatán. México. https://bit.ly/3uIs8dR

Diario Oficial de la Federación. Declaratoria de Emergencia. (2020b). DOF:15/06/2020. Quintana Roo. México. https://bit.ly/3wOWQ6N.

Diario Oficial de la Federación. Declaratoria de Emergencia. (2020c). DOF: 16/06/2020. Campeche. México. https://bit.ly/3dXxLOn

El Financiero. (2020). “Cristóbal” causa afectaciones en Campeche y Quintana Roo; Yucatán declara alerta amarilla. El Financiero. https://bit.ly/3sdQuuq.

Farfán, M. L., Prieto, R., Martínez-Sánchez, J., y Padilla, R. (2015). Ciclones tropicales y su influencia en México. En: Conviviendo con la Naturaleza. El problema de los Desastres asociados a Fenómenos Hidrometeorológicos y Climáticos en México. Cavazos, T.

México. 49-74. https://bit.ly/2Q2Tj4d.

García, E. (1997). Carta de Climas. Escala 1: 1000 000. CONABIO/Estadigrafía. México.

GOES. (2020). Imagen del GOES 16 Banda 13. https://bit.ly/3sjVBsM.

Instituto Nacional de Estadística y Geografía. (2010). Áreas Geoestadísticas Municipales, 2010. Escala: 1:250 000. Aguascalientes, Ags., México. https://bit.ly/3vRWtar.

Instituto Nacional de Estadística y Geografía. (2019). Carta Topográfica Digital escala 1:250 000. https://bit.ly/3o43QZy.

Instituto Nacional de Estadística y Geografía. (2020). Censo de Población y Vivienda 2020. México. https://bit.ly/3o6dsms.

Instituto Nacional de Estadística y Geografía. (2020b). Carta Hipsométrica Digital escala 1:1 000 000. https://bit.ly/3o43QZy. 
Jiménez, M., Matías L., Fuentes O., y Prieto, R. (2007). Ciclones tropicales. Fascículo CENAPRED-Secretaría de Gobernación. 55. https://bit.ly/3uZNVhp.

Landsea, C. (2018). Total and Average Number of Tropical Cyclones by Month (1851-2017). (Contributor from the NHC) aoml.noaa.gov. National Oceanic and Atmospheric Administration, Atlantic Oceanographic and Meteorological Laboratory. Archivado desde el original el 1 de septiembre de 2018. https://bit.ly/2PYi4i4

Landsea, C. W., Feuer, S., Hagen, A., Glenn, D., Anderson, N., Sims, J., Perez, R. y Chenoweth, M. (2011). A reanalysis of the 1921 to 1930 Atlantic Hurricane Database. Journal of Climate, 25, 865-885. https://doi.org/10.1175/JCLI-D-11-00026.1

Lansdea, C.W. (2007). Counting Atlantic tropical cyclones back to 1900. Eos, 88(18), 197-208. https://doi.org/10.1029/2007EO180001

López, E. (1973). Estudio Geológico de la Península de Yucatán. Gerencia de Exploración Pemex, 25-76. https://bit.ly/3wSjV8H.

Luces del Siglo. (2020). Estima Yucatán daños de Cristóbal en 4 mil mdp. Luces del Siglo. https://bit.ly/3a6hfL5.

Lugo Hub, J. Aceves-Quesada, F. J. y Espinasa Pereña, R. (1992). Rasgos geomorfológicos mayores de la Península de Yucatán. Revista Instituto de Geología Universidad Nacional Autónoma de México, 10(2), 143-150.

Lupo, R. A., Latham K. T., Magill H. T., Clark V. J., Melick J. C., y Market S. P. (2008). The interannual variability of hurricane activity in the Atlantic and East Pacific regions. National Weather Digest, 32(2), 119-133. http://hdl.handle.net/10355/2423

Mosiño, A. P. y García, E. (1974). The Climates of México. En Climates of North America, World Survey of Climatology (11 ed., 345-390). Amsterdam, Holanda: Elsevier Publ. Co.

National Hurricane Center (NHC-NOAA). (2020). National Hurricane Center and Central Pacific Hurricane Center. https://www.nhc.noaa.gov/data/tcr/.

National Hurricane Center. (1988). Preliminary report Hurricane Gilbert 08-19 September 1988. https://bit.ly/2PZqPsb.

Neumann, C. J., Jarvinen B. R., McAdie C. J., y Elms J.D. (1993). Tropical Cyclones of the North Atlantic Ocean, 1871-1992, Prepared by the National Climatic Data Center, Asheville, NC, in cooperation with the NHC, Coral Gables, FL, 193pp.

Nick, E. (2010). Interpolación de la precipitación en la cuenca del río Mantaro. En: Silva-Vidal, Y. Memoria del Subproyecto "Pronóstico estacional de lluvias y temperaturas en la cuenca del río Mantaro para su aplicación en la agricultura” 2007-2010. Instituto Geofísico del Perú (pp 19-24). https://repositorio.igp.gob.pe/handle/20.500.12816/708. 
Oficina Nacional de Administración Oceánica y Atmosférica. (2009). Tropical cyclone of the North Atlantic Ocean, 1851-2006. Historical climatology series, 6-2, p. 243.

Oficina Nacional de Administración Oceánica y Atmosférica. (2020a). Hurricane season information. https://www.aoml.noaa.gov/hrd-faq/\#hurricane-season.

Oficina Nacional de Administración Oceánica y Atmosférica. (2020b). Estatus del Sistema de alerta del ENSO, boletín mayo 2020. https://bit.ly/3bilCmF.

Oficina Nacional de Administración Oceánica y Atmosférica. (2020c). Monthly Atlantic Tropical Weather Summary, ABNT30 KNHC 011137, TWSAT del 1 de Agosto de 2020. https://bit.ly/3uHcyQc.

Oficina Nacional de Administración Oceánica y Atmosférica. (2020d). Bulletin Tropical Depression Three Discussion 1. ZCZC MIATCDAT3 AL032020, TTAA00 400 PM CDT Mon Jun 01 2020. https://bit.ly/3tAR7ie.

Oficina Nacional de Administración Oceánica y Atmosférica. (2020e). Bulletin Post-Tropical Cyclone Cristobal Advisory Number 35. https://bit.ly/3hnHcdc.

Oficina Nacional de Administración Oceánica y Atmosférica. (2020f). Tropical Storm CRISTOBAL Advisory Archive. https://bit.ly/2Q8p7F2.

Oficina Nacional de Administración Oceánica, y National Hurricane Center. (2002). Tropical Cyclone Report Hurricane Isidore 14-27 September 2002. https://bit.ly/3ezIJLN.

Orellana, R., Espadas, C., Conde, C., y Gay, C. (2009). Atlas, Escenarios de Cambio Climático en la Península de Yucatán. Centro de Investigación Científica de Yucatán, A. C. Mérida, Yucatán. México.

Palco Noticias Zona maya. (2020). Perdidas por 1MDP dejó la tormenta "Cristóbal" en cultivos de papaya en Lázaro Cárdenas. Palco Noticias Zona maya. Recuperado de https://bit.ly/3mITVrD

Papin, P.P. , Bosart, L. F. and Torn, R. D. (2017). A Climatology of Central American Gyres. Mon. Wea. Rev., 145(5), 1983-2000.

Por Esto. (25 de septiembre de 2020a). Desciende el nivel del agua en comunidad de Hopelchen afectada por Cristóbal. Por Esto. https://bit.ly/3dZHSIV.

Por Esto. (2020b). Ganaderos de Hopelchén exigen revaloración de daños por la Tormenta Tropical Cristóbal. Por Esto. https://bit.ly/3mIZmXz.

Puerto, P. (2020a). Con afectaciones por "Cristóbal”, 3 mil 500 familias de Quintana Roo. Yucatán a La Mano. https://bit.ly/.

Puerto, P. (2020b). A seis semanas del paso de "Cristóbal” Sahcabchén sigue bajo el agua. Yucatán a La Mano. https://bit.ly/3tb5wCi. 
Rosengaus, M., Espinoza M., y Vázquez M. (2014). Atlas climatológico de ciclones tropicales en México. CENAPRED-IMTA.1-108. https://bit.ly/3dXEqIC.

Rosengaus, M, Arreguín-Cortés, F., Korenfeld Federman, D., y Rubio-Gutiérrez, H. (2016). Visión panorámica de las precipitaciones pluviales combinadas por los efectos de los ciclones tropicales Ingrid-Manuel. Tecnología y Ciencias del Agua, 7(6), 73-92.

Rosengaus, M., y Sánchez, J. (1990). Gilbert: ejemplo de huracanes de gran intensidad. Ingeniería Hidráulica en México. https://bit.ly/32dQBeF

Sánchez, Á. (Coord.). (2006). Conocimientos Fundamentales de Geografía. Vol. I, [en CD-ROM], México, UNAM/McGraw-Hill, Colección Conocimientos Fundamentales 2006. https://bit.ly/3hkWcsr.

Small, R. J. O., de Szoeke, S. P., y Xie, S. (2007). The Central American Midsummer Drought: Regional Aspects and Large-Scale Forcing. Journal of Climate, 20(19), 4853-4873. https://bit.ly/3g8vK4Q.

Secretaria de Marina. (2020). Clasificación de la precipitación acumulada en 24 horas para pronóstico a corto y mediano plazo. https://bit.ly/3wLTXDM

Servicio Meteorológico Nacional. (2020a). Pronóstico para la temporada de ciclones tropicales 2020. https://bit.ly/3uN5HnH

Servicio Meteorológico Nacional. (2020b). Etapas de evolución de un ciclón tropical. https://bit.ly/3a4Arss.

Tang, B. H., y Neelin J. D. (2004). ENSO Influence on Atlantic hurricanes via tropospheric warming. Geophysical Research Letters, 31, (L24204). https://doi.org/10.1029/2004GL021072.

Tribuna Campeche. (2020). Más de mil en albergues; daño al agro por 40 MDP. Tribuna Campeche. https://bit.ly/32pdw7b.

Organización de las Naciones Unidas para la Educación, la Ciencia y la Cultura. (2009). Global trends in water-related disasters: an insight for policymakers. 28p. UNESCO. https://bit.ly/3dViFJk.

Villicaña, F. J., Azpra, R. E., y Hernández, M. E. (2016). A climatography of Tropical Cyclones in North Eastern Pacific Ocean. Analele Stiintifice Ale Universitatii. "Alexandru Ioan Cuza" din Iasi, LXII(1), s. II-c. 